\title{
Power Control for SISO Interference Channel Networks Based on Successive Convex Approximation
}

\section{Ruina Mao}

Qufu Normal University

Jiguo Yu

Qufu Normal University

Anming Dong ( $\sim$ anmingdong@qlu.edu.cn )

Qilu University of Technology https://orcid.org/0000-0001-7470-5159

Yue Wang

Qilu University of Technology

Baogui Huang

Qufu Normal University

\section{Research}

Keywords: Interference channel, Power control, Successive convex approximation (SCA), Sum rate maximization

Posted Date: February 14th, 2020

DOl: https://doi.org/10.21203/rs.2.23552/v1

License: (c) (i) This work is licensed under a Creative Commons Attribution 4.0 International License. Read Full License 


\title{
RESEARCH
}

\section{Power Control for SISO Interference Channel Networks Based on Successive Convex Approximation}

\author{
Ruina $\mathrm{Mao}^{1}$, Jiguo $\mathrm{Yu}^{1,2,3}$, Anming Dong ${ }^{2 *}$, Yue Wang ${ }^{2}$ and Baogui Huang ${ }^{1}$
}

\begin{abstract}
Interference channel (IC) is a classical model used to characterize the effect of interference for many real-life communication systems. In this paper, we consider a sum rate maximization problem subject to maximum power restriction at each transmitter for a single-input single-output (SISO) IC network. The considered power control problem is typically a nonconvex problem which is hard to solve directly. We propose a solving algorithm for this power control problem based on successive convex approximation (SCA). Specifically, we first reformulate the original nonconvex objective function as the difference of two concave (D.C.) functions near a given point in the feasible region of the solution space. After that, we construct a convex substitute function for the D.C. objective by approximating its second concave function with the one-order Taylor expansion. The problem is further reformulated as an unconstrained problem by transforming the constraints as a barrier function. This unconstrained problem is then solved iteratively using Newton's method. We update the substitute function given the newly obtained solution, near which the one-order Taylor expansion is performed again. This process is then repeated until a smooth point is reached. Simulation results show the effectiveness of the proposed SCA-based power control algorithm.
\end{abstract}

Keywords: Interference channel; Power control; Successive convex approximation (SCA); Sum rate maximization

\section{Introduction}

Interference channel (IC) networks can be used to represent lots of wireless communication scenarios, where multiple transmitters simultaneously transmit to their intended receivers in the same frequency band (e.g., [1-5]). For example, the wireless signals generated from neighboring cells in a cellular system will interfering to each other and an IC network can be adopted to model this case.

Transmit power represents a key degree of freedom in the management of interference, energy and connectivity of a multiuser wireless communication system [6-11]. Power control helps to improve the efficiency of spectral reuse and the desirable user experience $[12,13]$. Since the achievable rate of an IC network is interference limited, it is necessary to control the transmit powers to tackle the problem of interference. However, power control is recognized as a

${ }^{*}$ Correspondence: anmingdong@qlu.edu.cn

${ }^{2}$ School of Computer Science and Technology, Qilu University of

Technology (Shandong Academy of Sciences), University Road, 250353, Jinan, Shandong, China

Full list of author information is available at the end of the article main challenge in wireless interference channel networks when the system throughput is expected to be optimized. This is because the transmit power of different users are coupled in the interference, which is further embodied in the signal to interference-plusnoise Ratios (SINR) on wireless links. The optimization to the transmit powers is thus a nonconvex problem, which is NP-hard in general [12,14-16]. Therefore, sophisticated algorithms are required to deal with the nonconvexity in order to find the solutions.

Traditionally, the Geometric Programming (GP) method is efficient and effective in finding a feasible solution for the sum rate maximization problem of IC networks [17]. The GP-based method is derived under the assumption of high SINR regime, where the SINR of each link is assumed much larger than 0dB. However, the high-SINR assumption is not valid when the interference links are strong. As a result, the solution obtained by the GP is far from optimum because of the existence of interference links [18].

Due to the ability to deal with nonconvexity, the successive convex approximation (SCA) techniques have 
gained widely attention in the communication ans signal processing field. The basic idea of SCA is to approximate the original nonconvex problem by a series of convex subproblems (also referred as surrogates), each of which is tractable much easier than the original problem (e.g., [19-23]). By successively solving the approximation problems, a smooth point can be finally obtained. Recently, the SCA techniques have been applied to wireless communication systems to gain performance improvement for a variety of scenarios, such as beamforming and transceiver designs (e.g., [22, 24, 25]), energy efficiency optimization (e.g., $[26,27])$ and spectrum management [28], etc. The SCA technique also sheds new lights on the power control optimization problems for wireless networks, such as the power control of device-to-device (D2D) networks in [29] and [30]. However, there is still little work in the literature that has applied the SCA-based techniques to solve this problem in the IC networks.

In this paper, we aim at applying the SCA techniques to solve the sum rate maximization problem under individual transmit power constraints in a single input single output (SISO) IC network. Recall that the problem of power control for the sum rate maximization is challenging since it is nonconvex due to the existence of inter-user interference.

To summarize, our contributions can be described as follows.

- We reformulate the original nonconvex objective as the difference of two concave (D.C.) functions. And we then construct a substitute for the D.C. function by replacing the subtracter function with its one-order Taylor expansion. This substitute constitutes a convex approximation for the original nonconvex.

- To solve the substitute problem, we further reformulate it as an unconstrained optimization problem by constructing a barrier function to remove the constraints. This unconstrained problem is then solved iteratively using the Newton's method, where the backtracking line search (BLS) is utilized to find proper step size and direction for solution updating.

- After obtaining the optimal solution for the unconstrained problem, we update the substitute function near the newly obtained solution and then repeat the approximating process until a smooth point is reached.

- Finally, we show the effectiveness of the proposed SCA-based algorithm for the considered power control problem as compared with GP-based algorithm through simulations.

The rest of the paper is organized as follows. In Section 2 , related work during the past decades is summarized. We present the system model and formulate the power control problem in Section 3. In Section 4.1, the GP method is reviewed and in Section 4.2, we derive the SCA-based solving algorithm. Simulation results are provided in Section 5, and we conclude our work in Section 6.

\section{Related work}

Power control of signals associated with multiple users in wireless communication systems plays an important role in improving the system throughput and quality of service. Countless schemes of power control have been extensively studied since 1990s for different interference-limited channel models and application scenarios. For example, power control schemes for satisfying the SINR quality of service (QoS) requirements of IC networks were studied in [31] and [32]. Paper [33] and [34] studied power control schemes for ad hoc networks. Power control methods for multiple-hop interference channels were studied in [35]. In [36], power control schemes for multiple access (MAC) channels were proposed. Paper [37] proposed a new exponential power allocation scheme in the one-side interference channel relay network, whose nodes transmit signals by an exponential function of the maximum transmitted power with an exponential power allocation coefficient. Recently, new power control methods combined with machine learning techniques have also been proposed. For example, paper [16] proposed a deep neural network (DNN) based power control method to solve the nonconvex sum rate optimization problem for a fading multi-user interference channel.

In general, the design logic of these works can be classified as two kinds. The first one is to design the power control schemes to guarantee a target QoS level measured by the SINR or achievable rate to the users, and the other is to maximize the throughput given the available power and spectrum resources. Nevertheless, the power control optimization problems for IC networks are general nonconvex [18,38], and a closed-form solution can not be derived. Since each user's performance depends not only on its own power allocation but also on the power allocation of all other users, the power control problems in IC networks involve a performance tradeoff among different users. Consequently, the works on exploring the achievable rate region and developing heuristic algorithms are proposed in the literature to find achievable solutions to the problem.

In order to explore the achievable rate region, power control analyses based on game theory were studied in the earliest literature. Paper [6] formulated the multiuser power control problem of a frequency-selective interference channel as a non-cooperative Nash game. An iterative water-filling method was proposed to compute a Nash equilibrium solution. In [39], a power control game was studied by treating interference as noise 
at each receiver. It was shown that the fair and efficient operating point can be enforced through punishment strategies. Paper [40] characterized the equilibrium property a non-cooperative interference canceling and power control game in a 2-user Gaussian IC network. Although these algorithms may converge to a poor spectrum sharing strategy due to the problem's nonconvex nature, they provide a deep insight into the solution of the problem.

In order to circumvent the nonconvex structure, the GP-based power control methods for IC networks were investigated in [17]. But GP-based methods are derived under the assumption of high SINR regime, the performance of these methods may be far from optimal. In [41], it was shown that the optimal power allocation for a two-user IC network is binary, i.e., assigning full power to one user and shut down the other, or both users are allocated with full available powers. However, this result does not hold when the number of users is larger than two. Specifically, when the binary power control method is slightly inferior to that of the GP-based method.

Efforts have also been taken on finding the global optimal solutions for power control problems of IC networks. In [38] and [42], the power control problems were recast as D.C. programmings and the brunchand-bound algorithms were then utilized to find a global optimum. Nevertheless, the brunch-and-bound methods are computationally demanding since they perform global search over the entire solution space. To lessen complexity, paper [43] recasts the D.C. programming as a convex optimize problem by replacing the second term in the D.C. function with its firstorder approximation and developed an iterative algorithm to successively optimize the solution. It is noted that the convex software packages [44] was utilized to solve the convex optimization problem in [43], this may bring inconvenience to practical applications due to issues of compatibility and portability. Therefore, it is necessary to further develop dedicated algorithms for the power control problems of IC networks.

\section{System Model and Problem Formulation}

\subsection{Interference Channel Network Model}

We consider a SISO IC network scenario as shown in Fig. 1. There exists $K$ nodes in the network, and each node consists of one transmitter ( $\mathrm{Tx}$ ) and one receiver (Rx). Both the Tx and Rx of a node are equipped with single antenna. The $\mathrm{Tx}$ of a node transmit dedicated information to its paired Rx. We assume all nodes sharing the same frequency band and thus the information of one nodes constitutes interference to the Rxs of other pairs.

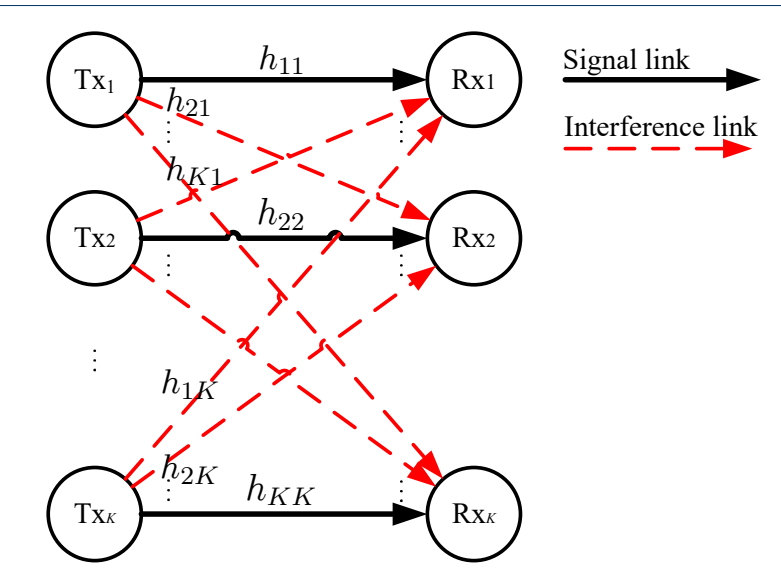

Figure 1 System model of the $K$-user SISO IC network.

Let $\mathcal{K}=\{1,2, \ldots, K\}$ denote the set of users, the signal observed by the $k$-th $\mathrm{Rx}$ is written as

$$
y_{k}=h_{k k} x_{k}+\sum_{j=1, j \neq k}^{N} h_{k j} x_{j}+n_{k}, \forall k \in \mathcal{K},
$$

where $x_{j}$ denotes the transmitted signal from the $j$-th Tx, $h_{k j}$ denotes the channel state information (CSI) from $\operatorname{Tx} j$ to $\mathrm{Rx} k . n_{k}$ is the zero-mean additive white Gaussian noise (AWGN) at the $k$-th Rx, which is independent identically distributed (i.i.d.) complex Gaussian distributed with $n_{k} \sim \mathcal{C N}\left(0, \sigma^{2}\right), \forall k \in \mathcal{K}$.

Without loss of generality, we assume the channels $h_{k j}, \forall k, j \in \mathcal{K}$ follow the Rayleigh block-fading channel model, where the channel parameters are constant within a frame and changes randomly between successive frames $[4,45]$.

The SINR at the $k$-th $\mathrm{Rx}$ is then calculated as

$$
\gamma_{k}=\frac{\left|h_{k k}\right|^{2} p_{k}}{\sum_{j=1, j \neq k}^{N}\left|h_{k j}\right|^{2} p_{j}+\sigma^{2}}, \forall k \in \mathcal{K},
$$

where $p_{j}=\mathbb{E}\left[x_{j}^{2}\right]$ denotes the transmit power of the $j$ th $\mathrm{Tx}, \forall j \in \mathcal{K}$. The achievable rate of the $k$-th node is denoted as

$$
R_{k}(\mathbf{p})=\log _{2}\left(1+\gamma_{k}\right)
$$

where $\mathbf{p} \triangleq\left[p_{1}, p_{2}, \ldots, p_{K}\right]^{T}$ is the vector constructed by the transmit power of all node. The sum rate of the IC network is then given by

$$
R(\mathbf{p})=\sum_{k=1}^{N} R_{k}(\mathbf{p})
$$




\subsection{Problem Formulation}

It is well known that IC networks are interference limited, which is embodied by the ceiling effect of the achievable sum rate. In other words, the sum rate is not bound to increase along with the increase of transmit power. Thus, it is necessary to optimize the transmit power to improve sum rate and meanwhile save energy.

In this paper, aiming at maximizing the sum rate given the transmit power budget, we consider the following power control problem

$$
\begin{array}{ll}
\max _{\mathbf{p}} & R(\mathbf{p}) \\
\text { s.t. } & p_{k} \geq p_{k}^{\text {min }}, \\
& p_{k} \leq p_{k}^{\text {max }}, \forall k \in \mathcal{K},
\end{array}
$$

where $p_{k}^{\text {min }}$ and $p_{k}^{\max }$ represent the minimum required transmit power and the maximum allowed transmit power of the $k$-th Tx, respectively.

Since the logarithm functions in the objective are equivalent to differences of concave functions, problem (5) is nonconvex optimization problem. Finding a good solution to this problem is still challenging, despite some previous work has been conducted in literature. Among different methods, the traditional GPbased algorithm is the most wildly used one to obtain an approximation solution for the problem [17]. In the following section, after briefly reviewing the GP-based algorithm for the considered problem(5), we propose an SCA-based power control method for it.

\section{Methods}

In this section, we briefly review the traditional GPbased power control method, which is used as the performance benchmark for our work. After that, we present the proposed SCA-based solving method for the formulated problem (5), which is a sum rate maximization problem subject to individual transmit power constraints for SISO IC network. Specifically, to deal with the nonconvexity of the problem, we first reformulate the rate objective as a D.C. function, and then relax the second term of the D.C. function as concave using the first-order Taylor expansion. The original problem is now approximated by the concave surrogate. This convex constrained problem is further reformulated as an unconstrained convex problem through barrier method, which can be finally solved efficiently using Newton's method. After initiating from a feasible point, an effective solution can be finally obtained by successively applying this convex approximation process.

\subsection{Review of the GP-based Power Control Method}

A GP is a nonlinear nonconvex optimization problem, which can be reformulated as a nonlinear convex problem. Since a GP can be turned into a convex optimization problem, its global optimum can be obtained efficiently with polynomial time complexity. In this section, we apply the traditional geometric progranming (GP) method to find effective solution for problem (5).

In order to reformulate problem (5) as a GP, we focus on high SINR regime, where $\log _{2}\left(1+\gamma_{k}\right)$ can be approximated by $\log _{2} \gamma_{k}$. The sum rate is then approximately written as

$$
\begin{aligned}
R(\mathbf{p}) & =\sum_{k=1}^{K} \log _{2}\left(1+\gamma_{k}\right) \\
& \approx \sum_{k=1}^{K} \log _{2} \gamma_{k} \\
& =\log _{2} \prod_{i=1}^{N} \gamma_{k}
\end{aligned}
$$

After replacing the objective with (6), problem (5) can be approximated by the following problem

$$
\begin{array}{ll}
\min _{\mathbf{p}} & \prod_{i=1}^{N} \gamma_{k}^{-1} \\
\text { s.t. } & p_{k}^{\text {min }} \leq p_{k} \leq p_{k}^{\text {max }}, \forall k \in \mathcal{K} .
\end{array}
$$

Problem (7) is a GP, which can be solved effectively using the disciplined convex optimization tools, such as the CVX [44].

Although the optimal solution of the GP (7) can be obtained, it may be far from optimum for the original problem (5) as indicated by literature [18], since the two problem is not exactly the same due to the approximation in (6). To overcome this drawback, we propose a novel solving algorithm for problem (5) based on SCA in the following section.

\subsection{SCA-based Transmit Power Control}

The GP-based algorithm is effective in obtain a feasible solution for the considered problem (5), but its optimality is not exactly known since the approximated problem (6) is not absolutely equivalent to (5), especially when the observed SINRs are not high enough [17].

In the following, we develop an SCA-based solving algorithm to find the solution of (5). The basic idea of the SCA is to approximate the original nonconvex problem by a series of convex subproblems (also referred as surrogates), each of which is tractable much 
easier than the original problem. By successively updating the surrogates using the solution of the last iteration, a feasible solution can be obtained iteratively.

\subsubsection{Convex Approximation to the Objective Function}

To develop the SCA-based algorithm, we rewrite (4) as the form of D.C. program

$$
R(\mathbf{p})=\sum_{k=1}^{K} \log _{2}\left(1+\gamma_{k}\right)=l(\mathbf{p})-h(\mathbf{p})
$$

where

$$
l(\mathbf{p})=\sum_{k=1}^{K} \log _{2}\left(\sum_{j=1}^{K}\left|h_{k j}\right|^{2} p_{j}+\sigma^{2}\right)
$$

and

$$
h(\mathbf{p})=\sum_{k=1}^{K} \log _{2}\left(\sum_{j=1, j \neq k}^{K}\left|h_{k j}\right|^{2} p_{j}+\sigma^{2}\right) .
$$

The functions $l(\mathbf{p})$ and $h(\mathbf{p})$ are concave, but the difference of them (i.e., (8)) is neither convex nor concave.

According to the idea of SCA, a concave lower bound (i.e., the surrogate function) for the non-concave objective (8) should be constructed, given a feasible solution $\mathbf{p}^{\prime}$ of problem (5). We construct this lower bound by replacing $h(\mathbf{p})$ with its first-order Taylor approximation

$$
R\left(\mathbf{p}, \mathbf{p}^{\prime}\right)=l(\mathbf{p})-\bar{h}\left(\mathbf{p}, \mathbf{p}^{\prime}\right)
$$

where $\bar{h}\left(\mathbf{p}, \mathbf{p}^{\prime}\right) \triangleq h\left(\mathbf{p}^{\prime}\right)-\nabla h\left(\mathbf{p}^{\prime}\right)\left(\mathbf{p}-\mathbf{p}^{\prime}\right)$ is the firstorder Taylor's expansion of the $h(\mathbf{p})$ near the solution $\mathbf{p}^{\prime} . \nabla h\left(\mathbf{p}^{\prime}\right)$ is the gradient of the $R(\mathbf{p})$ at $\mathbf{p}^{\prime}$, the $k$-th element of which can be derived as

$$
\begin{aligned}
\nabla_{k} h\left(\mathbf{p}^{\prime}\right) & =\frac{\partial h\left(\mathbf{p}^{\prime}\right)}{\partial p_{k}^{\prime}} \\
& =\frac{1}{\ln 2} \sum_{i=1, i \neq k}^{N} \frac{\left|h_{i k}\right|^{2}}{\sum_{j=1, j \neq i}^{K}\left|h_{i j}\right|^{2} p_{j}^{\prime}+\sigma^{2}}
\end{aligned}
$$

The surrogate function (11) is concave. Moreover, it is easy to know that the first-order Taylor's expansion is an upper bound of $h(\mathbf{p})$, i.e.,

$$
h(\mathbf{p}) \leq h\left(\mathbf{p}^{\prime}\right)+\nabla h\left(\mathbf{p}^{\prime}\right)\left(\mathbf{p}-\mathbf{p}^{\prime}\right)
$$

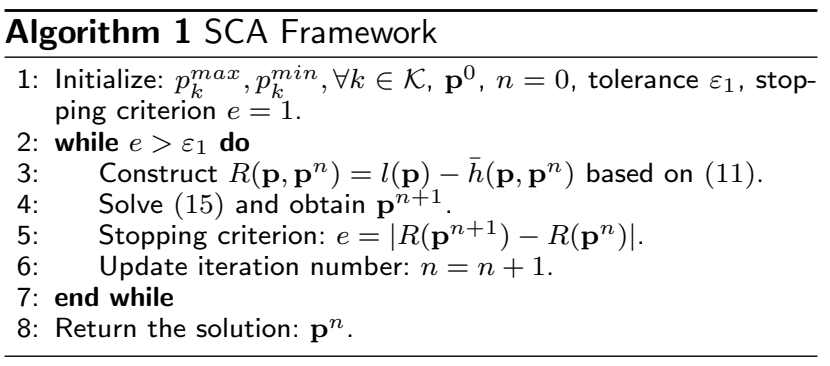

After combining (8), (11) and (13), we can obtain the following observation

$$
\begin{aligned}
R(\mathbf{p}) & =l(\mathbf{p})-h(\mathbf{p}) \\
& \geq l(\mathbf{p})-\left(h\left(\mathbf{p}^{\prime}\right)+\nabla h\left(\mathbf{p}^{\prime}\right)\left(\mathbf{p}-\mathbf{p}^{\prime}\right)\right) \\
& =l(\mathbf{p})-h\left(\mathbf{p}^{\prime}\right)-\nabla h\left(\mathbf{p}^{\prime}\right)\left(\mathbf{p}-\mathbf{p}^{\prime}\right) \\
& =R\left(\mathbf{p}, \mathbf{p}^{\prime}\right),
\end{aligned}
$$

which shows that the surrogate is a lower bound of the original function. The two functions are tangent at $\mathbf{p}^{\prime}$, i.e., $\left.R\left(\mathbf{p}, \mathbf{p}^{\prime}\right)\right|_{\mathbf{p}=\mathbf{p}^{\prime}}=R\left(\mathbf{p}^{\prime}\right)$. Therefore, $R\left(\mathbf{p}, \mathbf{p}^{\prime}\right)$ provides a tight low bound for the original objective function $R(\mathbf{p})$.

\subsubsection{Successive Convex Approximating Framework}

By replacing the original non-concave objective in problem (5) as the concave surrogate function (11), we obtain the following convex approximation problem to the original one

$$
\begin{array}{ll}
\min _{\mathbf{p}} & -R\left(\mathbf{p}, \mathbf{p}^{\prime}\right) \\
\text { s.t. } & p_{k} \geq p_{k}^{\text {min }}, \\
& p_{k} \leq p_{k}^{\max }, \forall k \in \mathcal{K} .
\end{array}
$$

Since problem (15) is convex, its solution can be obtained. We note that this solution is further used as the reference point for the next round of approximation. Specifically, after obtaining the new solution of problem (15), we update the value of $\mathbf{p}^{\prime}$ as this new solution, near which we construct a new surrogate function and thus a new approximated convex problem. This process is then run iteratively until a converged solution is found. We summarize this successive convex approximation solving framework for problem (5) in Algorithm 1.

The step 3 in Algorithm 1 is the convex approximation process and the step 4 achieves successive updating based on the new solution.

The successive convex approximating progress in Algorithm 1 must be convergent. To prove this point, we denote $\mathbf{p}^{n}$ as the optimal solution of (15) at the $n$-th 
iteration. Then, at the $(n+1)$-th iteration, the following relationship holds

$$
\begin{aligned}
R\left(\mathbf{p}^{n+1}\right) & =\left.R\left(\mathbf{p}, \mathbf{p}^{n}\right)\right|_{\mathbf{p}=\mathbf{p}^{n+1}} \\
& \geq\left. R\left(\mathbf{p}, \mathbf{p}^{n}\right)\right|_{\mathbf{p}=\mathbf{p}^{n}} \\
& =R\left(\mathbf{p}^{n}\right)
\end{aligned}
$$

which indicates that a series of non-decreasing solutions $\left\{\mathbf{p}^{n+1}\right\}$ of the original objective $R(\mathbf{p})$ can be acquired by iteratively solving the optimization (15). Since the value must be upper bounded by the optimal solution to (5), the sequence $\left\{\mathbf{p}^{n+1}\right\}$ always converges to a suboptimal solution.

We note that Algorithm 1 is just a sketchy framework to solve problem (5). In order to successfully apply Algorithm 1 in practice, the problem (15) should be solved. In the following, we develop an algorithm to solve it.

\subsubsection{Barrier Method}

Problem (15) is a convex optimization problem with inequality constraints. In this subsection, we develop an solving algorithm for this problem based on barrier method, which is a particular interior-point method [46]. The key idea of the barrier method is to approximately formulate the inequality constrained problem (15) as an unconstrained problem, which can be solved by Newton's method.

(1) Problem Reformulation Based on Logarithmic Barrier Function:

The first step of the barrier method is to recast the problem (15) as an unconstrained problem by making the inequality constraints implicit in the objective

$$
\begin{aligned}
\min _{\mathbf{p}} & -R\left(\mathbf{p}, \mathbf{p}^{\prime}\right)+\sum_{k=1}^{K} I_{-}\left(p_{k}^{\text {min }}-p_{k}\right) \\
& +\sum_{k=1}^{K} I_{-}\left(p_{k}-p_{k}^{\text {max }}\right)
\end{aligned}
$$

where $I_{-}(\cdot)$ is the indicator function defined as

$$
I_{-}(x)=\left\{\begin{array}{l}
0, x \leq 0 \\
\infty, x>0
\end{array}\right.
$$

Although problem (17) has no inequality constraints, its objective function is non-differentiable, which poses challenge to develop effective solving algorithm. In order to facilitate solving, we approximate the nondifferentiable indicator function $I_{-}(\cdot)$ by the differentiable logarithmic barrier function

$$
\hat{I}_{-}(x)=-\frac{1}{t} \log (-x), x \leq 0,
$$

where $t>0$ is a parameter that sets the accuracy of the approximation.

After replacing $I_{-}(x)$ with $\hat{I}_{-}(x)$ in $(17)$, we obtain the following approximation

$$
\begin{aligned}
\min _{\mathbf{p}} & -R\left(\mathbf{p}, \mathbf{p}^{\prime}\right)-\frac{1}{t} \sum_{k=1}^{K} \log \left(p_{k}^{\text {min }}-p_{k}\right) \\
& -\frac{1}{t} \sum_{k=1}^{K} \log \left(p_{k}-p_{k}^{\text {max }}\right) .
\end{aligned}
$$

The problem (19) is convex, since $\hat{I}_{-}(x)$ is convex and increasing in $x$.

To facilitate algorithm design, we rewrite problem (19) as the following unconstrained minimization problem

$$
\min _{\mathbf{p}} \varphi\left(\mathbf{p}, \mathbf{p}^{\prime}\right) \triangleq-t R\left(\mathbf{p}, \mathbf{p}^{\prime}\right)+\phi(\mathbf{p})
$$

where $\phi(\mathbf{p}) \triangleq-\sum_{k=1}^{K} \log \left(p_{k}-p_{k}^{\text {min }}\right)-\sum_{k=1}^{K} \log \left(p_{k}^{\max }-\right.$ $\left.p_{k}\right)$ is called the logarithmic barrier. The domain of $\phi(\mathbf{p})$ is the set of points that satisfy the power constraints strictly in (15). For any given positive value of $t$, the logarithmic barrier grows without bound if $p_{k} \rightarrow p_{k}^{\min }$ or $p_{k} \rightarrow p_{k}^{\max }, \forall k \in \mathcal{K}$.

\subsubsection{Optimality of Barrier Approximation}

We note that problem (20) is only an approximation of the original problem (15), and the accuracy of approximation is determined by the parameter $t$ [46]. Specifically, denote $\mathbf{p}^{*}$ and $\mathbf{p}^{*}(t)$ as the optimal solution of (15) and (20), respectively, then accuracy of the solution $\mathbf{p}^{*}(t)$ is bounded by

$$
R\left(\mathbf{p}^{*}(t), \mathbf{p}^{\prime}\right)-R\left(\mathbf{p}^{*}, \mathbf{p}^{\prime}\right) \leq \frac{2 K}{t}
$$

which shows that $\mathbf{p}^{*}(t)$ is not worse than $2 K / t$ suboptimal. This reveals that the quality of the approximation improves as the parameter $t$ grows, i.e., $\lim _{t \rightarrow \infty} \mathbf{p}^{*}(t)=\mathbf{p}^{*}$.

Nevertheless, when the parameter $t$ becomes large, the Hessian of $\varphi\left(\mathbf{p}, \mathbf{p}^{\prime}\right)$ varies rapidly near the boundary of the feasible set, which makes it difficult to minimize the function $R\left(\mathbf{p}, \mathbf{p}^{\prime}\right)+\frac{1}{t} \phi(\mathbf{p})$ by Newton's method. This problem can be circumvented by solving a sequence of problems of the form (20) with increasing values of $t$, and starting each Newton minimization at the solution of the problem for the previous value of $t$.

\section{(2) Barrier Method}

Based on the above analysis, the barrier method is established by solving a sequence of unconstrained 


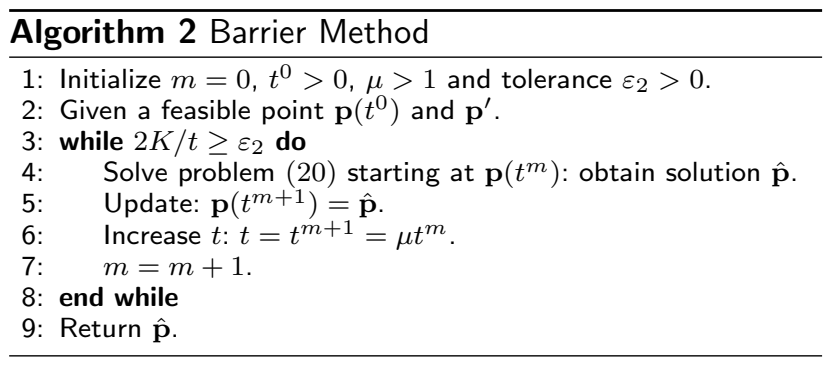

minimization problems shown by (20) with increasing values of $t$, using the last point found as the starting point for the next unconstrained minimization problem. The framework of the barrier method is summarized in Algorithm 2.

The step 4 of Algorithm 2 is also called a centering step. It is noted that any method for linearly constrained minimization can be used in step 4. Moreover, computing the solution of (20) exactly is not necessary, since inexact centering will still yield a sequence of points $\mathbf{p}\left(t^{m}\right)$ that converges to an optimal point as $t \rightarrow \infty$ [46]. In this work, we use the Newton's method to obtain the solution in step 4 .

\subsubsection{Newton's Method for the Unconstrained Problem}

Problem (20) can be efficiently solved through Newton's method. In Newton's method, solving an unconstrained or equality constrained problem is reduced to solving a sequence of quadratic problems [46].

(1) Newton Step

To develop the Newton's method, we write the second-order Taylor approximation of the objective function of (20) at $\mathbf{p}$ as

$$
\begin{aligned}
\hat{\varphi}\left(\mathbf{p}+\mathbf{v}, \mathbf{p}^{\prime}\right)= & \varphi\left(\mathbf{p}, \mathbf{p}^{\prime}\right)+\nabla \varphi\left(\mathbf{p}, \mathbf{p}^{\prime}\right)^{T} \mathbf{v} \\
& +\frac{1}{2} \mathbf{v}^{T} \nabla^{2} \varphi\left(\mathbf{p}, \mathbf{p}^{\prime}\right) \mathbf{v}
\end{aligned}
$$

Equation (22) is a convex quadratic function of $\mathbf{v}$. By setting its first-order derivative with respect to $\mathbf{v}$ as zero, $\hat{\varphi}\left(\mathbf{p}+\mathbf{v}, \mathbf{p}^{\prime}\right)$ is minimized when the following condition establishes

$$
\mathbf{v}=\boldsymbol{\delta}_{n t}\left(\mathbf{p}, \mathbf{p}^{\prime}\right) \triangleq-\left(\nabla^{2} \varphi\left(\mathbf{p}, \mathbf{p}^{\prime}\right)\right)^{-1} \nabla \varphi\left(\mathbf{p}, \mathbf{p}^{\prime}\right)
$$

$\boldsymbol{\delta}_{n t}\left(\mathbf{p}, \mathbf{p}^{\prime}\right)$ is called the Newton step, which can be added to the point $\mathbf{p}$ to minimize the second-order approximation of $g(\cdot)$ at $\mathbf{p}$.

Under the considered SISO IC network model, the $k$-th element of the gradient $\nabla \varphi\left(\mathbf{p}, \mathbf{p}^{\prime}\right)$ can be derived as

$$
\begin{aligned}
\nabla_{k} \varphi\left(\mathbf{p}, \mathbf{p}^{\prime}\right)= & \frac{\partial \varphi\left(\mathbf{p}, \mathbf{p}^{\prime}\right)}{\partial p_{k}} \\
= & -t \frac{1}{\ln 2}\left(\sum_{i=1}^{K} \frac{\left|h_{i k}\right|^{2}}{\sum_{j=1}^{K}\left|h_{i j}\right|^{2} p_{j}+\sigma^{2}}\right. \\
& \left.-\sum_{i=1, i \neq k}^{N} \frac{\left|h_{i k}\right|^{2}}{\sum_{j=1, j \neq k}^{K}\left|h_{k j}\right|^{2} p_{j}^{\prime}+\sigma^{2}}\right) \\
& -\frac{1}{p_{k}-p_{k}^{\text {min }}}+\frac{1}{p_{k}^{\text {max }}-p_{k}} .
\end{aligned}
$$

On the other hand, the element at the cross of the $l$-th row and the $k$-th column of the Hessian can be derived as

$$
\nabla_{l, k}^{2} \varphi\left(\mathbf{p}, \mathbf{p}^{\prime}\right)=\left\{\begin{array}{c}
t \frac{1}{\ln 2} \sum_{i=1}^{K} \frac{\left|h_{i k}\right|^{2}\left|h_{i l}\right|^{2}}{\left(\sum_{j=1}^{K}\left|h_{i j}\right|^{2} p_{j}+\sigma^{2}\right)^{2}}, \\
\forall l, k \in \mathcal{K}, l \neq k, \\
t \frac{1}{\ln 2} \sum_{i=1}^{K} \frac{\left|h_{i k}\right|^{2}\left|h_{i l}\right|^{2}}{\left(\sum_{j=1}^{K}\left|h_{i j}\right|^{2} p_{j}+\sigma^{2}\right)^{2}}, \\
+\frac{1}{\left(p_{l}-p_{l}^{\text {min }}\right)^{2}}+\frac{1}{\left(p_{l}^{\text {max }}-p_{l}\right)^{2}}, \\
\forall l, k \in \mathcal{K}, l=k .
\end{array}\right.
$$

\section{(2) Newton Decrement}

After substituting (23) into (22), the minimum of the second-order approximation $\hat{\varphi}\left(\mathbf{p}+\mathbf{v}, \mathbf{p}^{\prime}\right)$ can be written as

$$
\inf _{\mathbf{v}} \hat{\varphi}\left(\mathbf{p}+\mathbf{v}, \mathbf{p}^{\prime}\right)=\varphi\left(\mathbf{p}, \mathbf{p}^{\prime}\right)-\frac{1}{2} \lambda^{2}\left(\mathbf{p}, \mathbf{p}^{\prime}\right),
$$

where $\lambda\left(\mathbf{p}, \mathbf{p}^{\prime}\right) \triangleq\left(-\nabla \varphi\left(\mathbf{p}, \mathbf{p}^{\prime}\right)^{T} \boldsymbol{\delta}_{n t}\left(\mathbf{p}, \mathbf{p}^{\prime}\right)\right)^{1 / 2}$ is called the Newton decrement, and the last term in (26) reflects the difference between $\hat{\varphi}\left(\mathbf{p}, \mathbf{p}^{\prime}\right)$ and the minimum of the second-order approximation. Therefore, it can be used as a stopping criterion for the iterating.

\section{(3) Newton's Method}

In practice, the Newton step is used to update the solution based on the following equation

$$
\mathbf{p}^{n+1}=\mathbf{p}^{n}+s \boldsymbol{\delta}_{n t}\left(\mathbf{p}^{n}, \mathbf{p}^{\prime}\right)
$$

where $\mathbf{p}^{n}$ is the solution obtained after the $n$-th iteration. $s>0$ is the step size, which determines where the next iterate will be along the line $\mathbf{p}^{n}+s \boldsymbol{\delta}_{n t}$.

The convergence of the Newton's method is determined by the step size $s$. A simple way is to set it as a fixed positive value. But this casual setting of the step size may be too small or too long. A small step size will make the convergence slow, while a too long step size may cause divergence of the algorithm. In order to ensure fast convergence, the line 

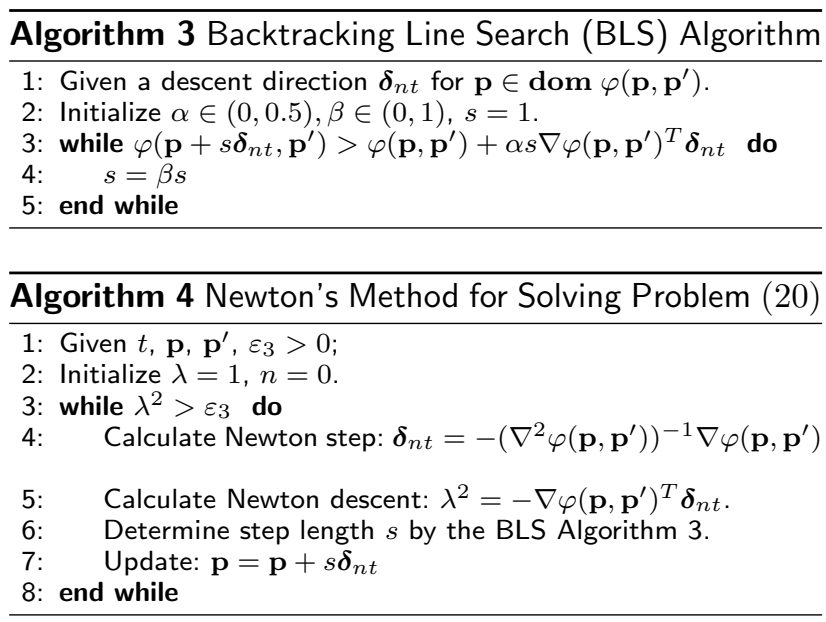

search methods, e.g., the exact and inexact line search methods, can be used to select a value for the step size $s$. In this work, we adopt the inexact method, which choses the step length to approximately minimize $\varphi\left(\mathbf{p}, \mathbf{p}^{n}\right)$ along the line $\mathbf{p}^{n}+s \boldsymbol{\delta}_{n t}, s>0$. Specifically, we adopt the backtracking line search (BLS) algorithm given by Algorithm 3. It depends on two constants $\alpha$ and $\beta$ with $0<\alpha<0.5$ and $0<\beta<1$. The algorithm starts with unit step size and then reduces it by the factor $\beta$ until the stopping condition $\varphi\left(\mathbf{p}+s \boldsymbol{\delta}_{n t}, \mathbf{p}^{\prime}\right) \leq \varphi(\mathbf{p})+\alpha s \nabla \varphi\left(\mathbf{p}, \mathbf{p}^{\prime}\right)^{T} \boldsymbol{\delta}_{n t}$ holds.

The Newton's method is then summarized in Algorithm 4.

\subsubsection{The Proposed SCA-based Power Control Algorithm}

By combining the Algorithm 4 with the SCA framework shown by Algorithm 1, we propose the SCAbased power control method for the sum rate maximization problem of the consider SISO IC network, which is given by Algorithm 5 .

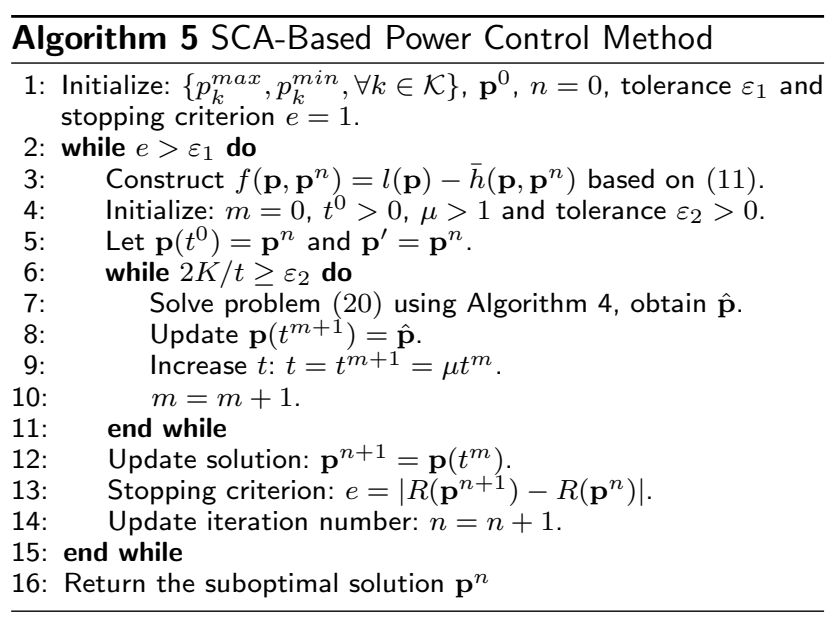

Table 1 Simulation parameter settings.

\begin{tabular}{ccc}
\hline Parameter & Value & Comments \\
\hline$N$ & 3 & Number of users \\
$\sigma^{2}$ & 1 & Normalized noise power \\
$\varepsilon_{1}$ & $10^{-5}$ & Tolerance of SCA iterating in Algorithm 5 \\
$\varepsilon_{2}$ & $10^{-4}$ & Tolerance for the iterating of Barrier method \\
$\varepsilon_{3}$ & $10^{-4}$ & Tolerance for the iterating of Newton's method \\
$t^{0}$ & 1 & Initial value of $t$ for the Barrier method \\
$\mu$ & 1.1 & Scaling factor for $t$ of the Barrier method \\
$\alpha$ & 0.1 & Parameter for BLS algorithm \\
$\beta$ & 0.2 & Parameter for BLS algorithm \\
\hline
\end{tabular}

\subsubsection{Complexity Analysis}

The proposed SCA-based power control algorithm consists of three loops. We denote the iteration numbers of the three tiers as $K_{1}, K_{2}, K_{3}$ respectively. The complexity of the proposed Algorithm 5 is related to a number of factors, including the tolerance $\varepsilon_{1}, \varepsilon_{2}$ and $\varepsilon_{3}, t^{0}, \mu$ and the dimension of the constraints $2 K$, etc. For the barrier method, the desired accuracy $\varepsilon_{2}$ is achieved after about $K_{2} \approx\left\lceil\frac{\log \left(2 K /\left(\varepsilon_{2} t^{0}\right)\right)}{\log \mu}\right\rceil$ centering steps [46]. For the Newton's method, the number of iterations is approximated by $K_{3} \approx \frac{f\left(\mathbf{p}^{0}\right)-f\left(\mathbf{p}^{*}\right)}{\varepsilon_{3}}+6$. The main calculation complexity comes from the step 4 of Algorithm 4, which is of $\mathcal{O}\left(K^{3}\right)$. Therefore, the total computation complexity of the proposed algorithm is about $\mathcal{O}\left(K_{1} K_{2} K_{3} K^{3}\right) \approx \mathcal{O}\left(K^{3} \log K\right)$.

\section{Results and Discussion}

In this section, numerical results are provided to validate the performance of the proposed power control algorithm. In the simulation, the channel parameters $h_{k j}, \forall k, j$ are generated following the i.i.d. complexity Gaussian distribution with zero mean and unit variance. To facilitate performance comparison, all the simulations are based on on the following randomly generated channel matrix

$$
\left[\left|h_{k j}\right|^{2}\right]_{\forall k, j}=\left[\begin{array}{lll}
2.1739 & 0.3841 & 1.4587 \\
3.4177 & 0.7276 & 4.9367 \\
2.6844 & 0.6578 & 2.3264
\end{array}\right] .
$$

The key parameters used in the proposed SCA-based algorithm are listed in Table 1. To facilitate performance comparison, we consider a 3-user IC network. Without loss of generality, we normalize the power of noise, i.e., $\sigma^{2}=1$. In this case, the transmit power represents the signal-to-noise ratio (SNR).

\subsection{Convergence Performance}

In order to demonstrate the convergence performance of the proposed algorithm, we first consider a scenario where the maximum available transmit powers of the users are different, i.e., $p_{1}^{\max }=20 \mathrm{~dB}, p_{2}^{\max }=15 \mathrm{~dB}$ 
and $p_{3}^{\max }=10 \mathrm{~dB}$. It is also assumed that the required minimum transmit powers are also different, i.e., $p_{1}^{\min }=10 \mathrm{~dB}, p_{2}^{\min }=8 \mathrm{~dB}$ and $p_{3}^{\min }=6 \mathrm{~dB}$.

It is worth noting that special attention should be paid to the setting of the parameters $\alpha$ and $\beta$ in the BLS algorithm and $\beta$ for the Barrier method. In our simulations, we find that relative small values are preferred to avoid the bad condition number of the Hessian matrix in (25), which may result in problems when calculating its inverse matrix.

Fig. 2, Fig. 3 and Fig. 4 show the convergence property of the proposed SCA-based power control algorithm under the parameter setting given by Table 1 . In the simulations, we start the algorithm using three different initializing methods for the transmit power.

- Minimum required power: initialize the transmit powers by scaling of minimum required transmit powers, e.g., $\left\{p_{k}^{0}=1.1 p_{k}^{\text {min }}, \forall k \in \mathcal{K}\right\}$.

- Maximum allowed power: initialize the transmit powers by scaling of the maximum allowed values, e.g., $\left\{p_{k}^{0}=0.98 p_{k}^{\max }, \forall k \in \mathcal{K}\right\}$.

- Randomly initialized power: initialize the transmit powers by values ranging from $p_{k}^{\min }$ to $p_{k}^{\max }, \forall k \in$ $\mathcal{K}$, e.g., $\left\{p_{1}^{0}=84.8070, p_{2}^{0}=29.6569, \quad p_{3}^{0}=\right.$ $7.8622\}$.

It can be observed from the figures that the algorithm converges after several iterations, no matter which initializing method is adopted. This means that its convergence speed is very fast. It is also noticed in the figures that the random initialized method converges the fastest.

In addition to the convergence speed, some interesting phenomenons are also demonstrated in Fig. 2,

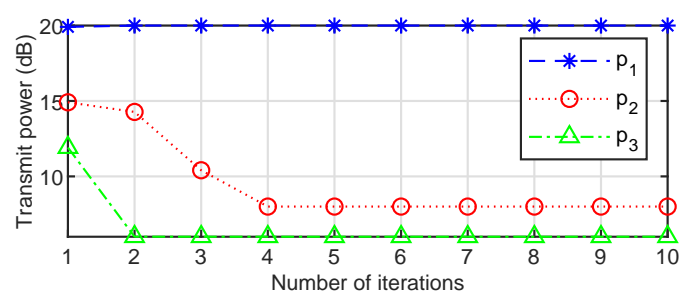

(a) Transmit power

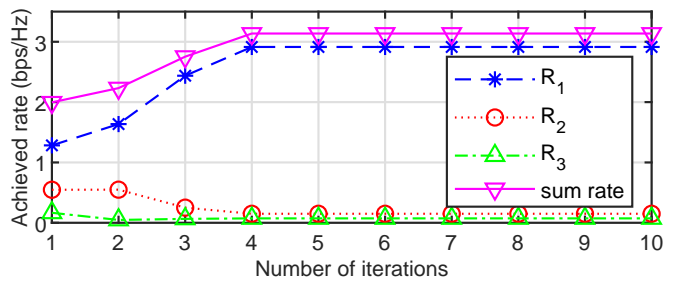

(b) Achieved rate

Figure 3 Convergence of the SCA-based algorithm with $\left\{p_{k}^{0}=0.98 p_{k}^{\max }, \forall k \in \mathcal{K}\right\}$.

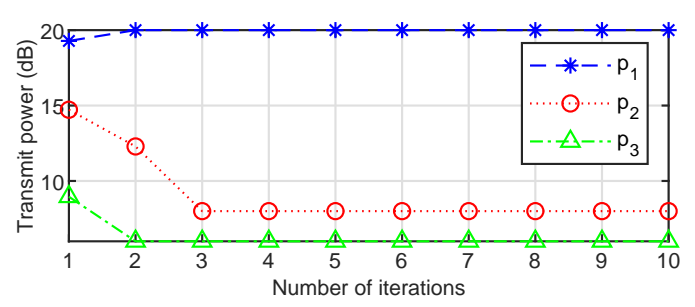

(a) Transmit power

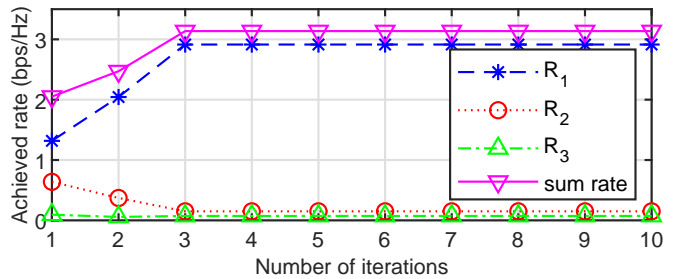

(b) Achieved rate

Figure 4 Convergence of the SCA-based algorithm with $\left\{p_{1}^{0}=84.8070, p_{2}^{0}=29.6569, p_{3}^{0}=7.8622\right\}$.

Fig. 3 and Fig. 4. Specifically, the optimized powers fall in the given power range between $p_{k}^{\min }$ to $p_{k}^{\max }$, which shows that the power constraints in (15) are satisfied. Moreover, it is noticed that the rate achieved by user 1 increases along with iterating, while the rates of the other two users decrease. Although some users decrease their rates, the sum rate of the whole network will increase along with iterating. It can be also observed that the optimized transmit power of user 1 reaches the maximum allowed value (i.e., 20dB), while user 2 and user 3 are allocated with the minimum required powers (i.e., $8 \mathrm{~dB}$ and $6 \mathrm{~dB}$, respectively). These observations imply that some users sacrifice 


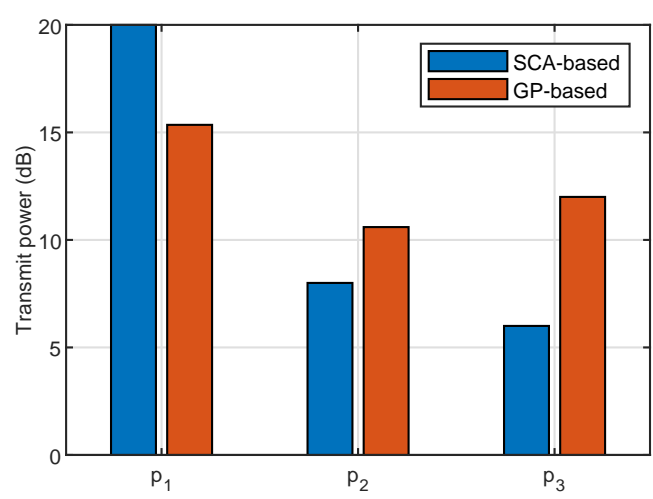

Figure 5 Comparison of the optimized transmit powers of the proposed SCA-based algorithm and the GP-based algorithm.

their achievable rate to alleviate the interference such that the sum rate of the whole network is benefited. All these phenomenons show the effectiveness of the proposed SCA-based power control algorithm in solving the sum rate maximization problem (5).

\subsection{Performance Comparison with GP-based Algorithm} In Fig. 5, we compare the power optimization results of the proposed SCA-based algorithm and GP-based algorithm for the 3 -user IC network. The results show that the proposed SCA-based algorithm allocates the maximum allowed power to user 1 and the minimum required powers to the user 2 and user 3, respectively. While the powers allocated by the GP-based algorithm fall in the ranges between the minimum required values and maximum allowed values. It can also be observed that the difference in the optimized transmit powers of the GP-based algorithm is not less significant than that of the SCA-based method.

The impact of these power allocation results on the achieved data rates is further compared in Fig. 6. It can be observed that the sum rate achieved by the GP-based method is significantly less than that of the SCA-based method, although the rates of use 2 and user 3 for the former are higher than that of the later. This further validates the judgment on the performance of the GP-based algorithm stated in literature [18], i.e., the solution obtained by the GP is far from optimum.

\subsection{Optimality Analysis}

Under the above-described system setting, more simulations show that the proposed SCA-based algorithm can achieve the same sum rate after converged, no matter which initializing method is adopted. This implies that the proposed SCA-based algorithm solves

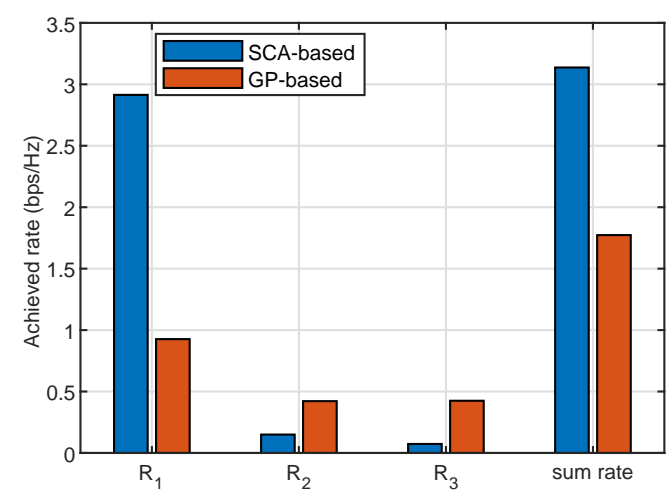

Figure 6 Comparison of the achieved data rates of the proposed SCA-based algorithm and the GP-based algorithm.

the original problem optimally. However, our further investigation shows that the proposed SCA-based algorithm may stick in local optimal solution under some other system settings. To reveal this point, we consider a different scenario where all users are configured as the same maximum allowed transmit power, i.e., $p_{k}^{\max }=p^{\max }, \forall k \in \mathcal{K}$, and the same minimum allowed transmit power as zero, i.e., $p_{k}^{\min }=0, \forall k \in \mathcal{K}$.

For the following, the greedy power control method, the non-cooperative scheme and the GP-based scheme are adopted as performance benchmarks. For the greedy scheme, only the user with the highest channel gain is activated. While in the non-cooperative scheme, all users transmit using the maximum allowed power.

By setting the $p^{\max }=20 \mathrm{~dB}$, we run the SCA-based method for 100 trials with randomly initializing. The simulation results are shown in Fig. 7. It can be observed that the greedy scheme achieves the optimal solution. The non-cooperative scheme achieves the lowest sum rate, since the high interference limits the achievable rate. The SCA-based algorithm can obtain three different solutions, including the optimal solution. The GP-based algorithm performs slightly better than the non-cooperative scheme, but far worse than the SCA-based scheme.

In oder to gain deeper insight into the performance of the proposed method, we list the achieved sum rate and the allocated powers of different schemes in Table 2. For the greedy scheme, the maximum power is allocated to user 3 , and the other two users are inactive, and the maximum sum rate $7.8681 \mathrm{bps} / \mathrm{Hz}$ is achieved. For the SCA-based scheme, the first solution achieves a sum rate of $6.2047 \mathrm{bps} / \mathrm{Hz}$ by activating user 2. Similarly, the second and third solutions of the SCA-based scheme achieve rates of $7.7707 \mathrm{bps} / \mathrm{Hz}$ and $7.8681 \mathrm{bps} / \mathrm{Hz}$ by activating user 1 and user 3, respectively. 


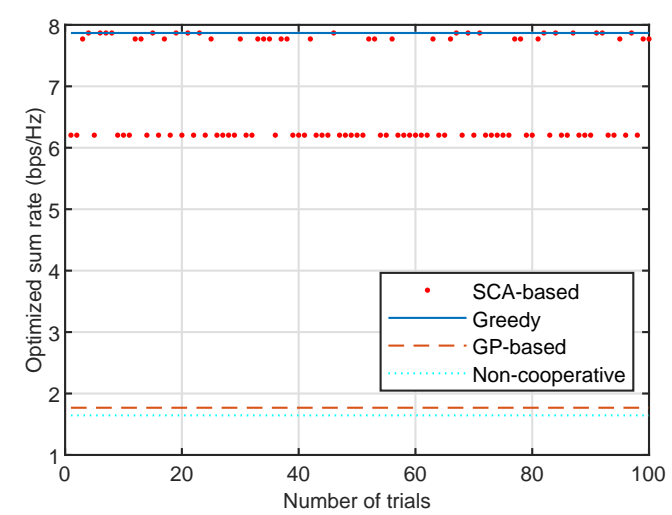

Figure 7 Sum rate achieved by the SCA-based algorithm over 100 randomly feasible initial points with $p_{k}^{\max }=20 \mathrm{~dB}$ and $p_{k}^{\text {min }}=0, \forall k \in \mathcal{K}$.

Table 2 Comparison of the rate and power allocation of different solutions

\begin{tabular}{rrrrr}
\hline Scheme & rate & $p_{1}$ & $p_{2}$ & $p_{3}$ \\
\hline SCA-based & 6.2047 & 0.0000 & 99.9988 & 0.0000 \\
SCA-based & 7.7707 & 99.9988 & 0.0000 & 0.0000 \\
SCA-based & 7.8681 & 0.0000 & 0.0000 & 99.9988 \\
Greedy & 7.8681 & 0.0000 & 0.0000 & 100.0000 \\
GP-based & 1.7685 & 100.0000 & 34.9255 & 51.8279 \\
Non-cooperative & 1.6445 & 100.0000 & 100.0000 & 100.0000 \\
\hline
\end{tabular}

Finally, in Fig. 8, we show the achieved sum rate of different algorithms as a function of the pseudo signal to noise ratio, which is defined as $\mathrm{SNR}^{\max } \triangleq \frac{p^{\text {max }}}{\sigma^{2}}$. Considering that the proposed SCA-based algorithm may be stuck in a locally optimal solution, we run it for 10 times at each SNR ${ }^{\max }$, and choose the best solution. It can be observed in the figure that the proposed SCA-based algorithm achieves similar performance as the greedy scheme when $\mathrm{SNR}^{\text {max }}$ is higher than $0 \mathrm{~dB}$, and performs far better than the GP-based scheme. When $\mathrm{SNR}^{\max }$ is lower than $0 \mathrm{~dB}$, the greedy scheme performs worse than other schemes, and the proposed SCA-based algorithm achieve a similar performance as the GP and non-cooperative scheme. It is worth mentioning that at low SNR regime, treating interference as noise is optimal according to information theory [47]. This is why the non-cooperative scheme performs superior to the greedy scheme in low SNR regime (e.g., SNR ${ }^{\max }<-8 \mathrm{~dB}$ ). Through these comparisons, we can conclude that the proposed SCA-based scheme is effective in finding at least a suboptimal solution at any SNR regime.

In summary, the proposed SCA-based power control algorithm is effective in finding the solution of sum rate maximization problem with peruser power constraint. It has the merits of fast convergence and acceptable computational complexity, and applicable at any SNR

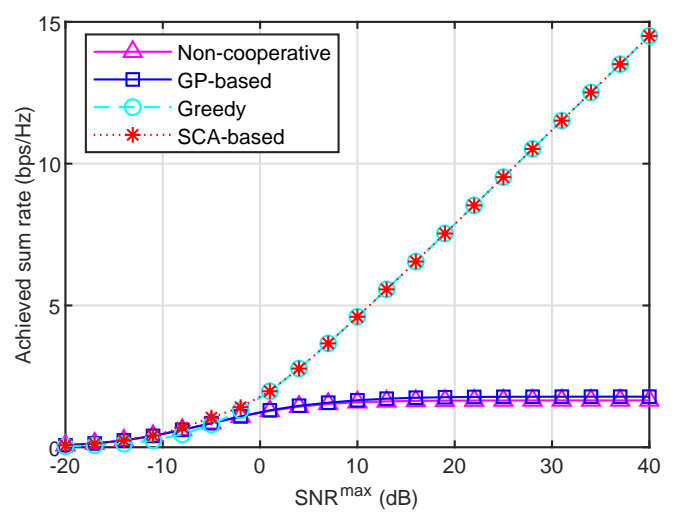

Figure 8 Sum rate versus the pseudo signal to noise ratio $\mathrm{SNR}^{\max }$ for different power control schemes with $p_{k}^{\max }=20 \mathrm{~dB}$ and $p_{k}^{\text {min }}=0, \forall k \in \mathcal{K}$.

regime. It is thus a candidate scheme for power control of interference channel networks.

\section{Conclusion}

We studied a sum rate maximization power control method for a single-input single-output (SISO) interference channel network. We proposed an SCA-based solving algorithm for this nonconvex power control problem. The key technique for this method is to reformulate the original nonconvex objective function as the difference of two concave functions and then relax it as convex by approximating the second concave function with its one-order Taylor expansion. The barrier method was applied to further recast the convex substitute as an unconstrained convex problem, which was solved efficiently using Newton's method. A feasible solution was finally obtained by updating the substitutes in a successive way. Simulation results showed the effectiveness of the proposed SCA-based power control algorithm. In future research, the joint power control and beamforming/transceiver designs based on SCA for more complicated multiple-input multiple-output(MIMO) IC networks should be further considered.

\section{Abbreviations}

SISO: Single-input single-output; IC: Interference channel; SCA: Successive convex approximation; D.C.: Difference of two concave; SINR: Signal to interference-plus-noise ratios; GP: Geometric programming; D2D:

Device-to-device; BLS: Backtracking line search; QoS: Quality of service; MAC: Multiple access; DNN: Deep neural network; SNR: Signal-to-noise ratio; MIMO: multiple-input multiple-output

Competing interests

The authors declare that they have no competing interests.

\section{Funding}

This work is partially funded by the National Natural Science Foundation of China (NSFC) under Grants 61701269, 61672321, 61832012, 61771289 and 
61373027, the Natural Science Foundation of Shandong Province under Grant ZR2017BF012.

\section{Author's contributions}

R. Mao and A. Dong made the theoretical derivations, designed the algorithms and analyzed the simulation results. B. Huang contributed in algorithm design. R. Mao and Y. Wang carried out the simulations. J. Yu supervised the research activity and guided the theoretical analysis.

\section{Author details}

${ }^{1}$ School of Information Science and Engineering, Qufu Normal University, Yantai Road, 276826, Rizhao, Shandong, China. ${ }^{2}$ School of Computer Science and Technology, Qilu University of Technology (Shandong Academy of Sciences), University Road, 250353, Jinan, Shandong, China. ${ }^{3}$ Shandong Provincial Key Laboratory of Computer Networks, Shandong Computer Science Center (National Supercomputer Center in Jinan), Xinluo Street, 250014, Jinan, Shandong, China.

\section{References}

1. Han, T., Kobayashi, K.: A new achievable rate region for the interference channel. IEEE Transactions on Information Theory 27(1) 49-60 (1981)

2. Etkin, R.H., David, N., Wang, H.: Gaussian interference channel capacity to within one bit. IEEE Transactions on Information Theory 54(12), 5534-5562 (2008)

3. Jafar, S.A., Fakhereddin, M.J.: Degrees of freedom for the MIMO interference channel. IEEE Transactions on Information Theory 53(7), 2637-2642 (2007)

4. Dong, A., Zhang, H., Yuan, D., Zhou, X.: Interference Alignment Transceiver Design by Minimizing the Maximum Mean Square Error for MIMO Interfering Broadcast Channel. IEEE Transactions on Vehicular Technology 65(8), 6024-6037 (2016)

5. Yu, J., Huang, B., Cheng, X., Atiquzzaman, M.: Shortest link scheduling algorithms in wireless networks under the SINR model. IEEE Transactions on Vehicular Technology 66(3), 2643-2657 (2017)

6. Yu, W., Ginis, G., Cioffi, J.M.: Distributed multiuser power control for digital subscriber lines. IEEE Journal on Selected Areas in Communications 20(5), 1105-1115 (2002)

7. Huang, B., Yu, J., Cheng, X., Chen, H., Liu, H.: SINR based shortest link scheduling with oblivious power control in wireless networks. Journal of Network and Computer Applications 77, 64-72 (2017)

8. Yu, D., Zou, Y., Yu, J., Cheng, X., Hua, Q.-S., Jin, H., Lau, F.C.: Stable local broadcast in multihop wireless networks under SINR. IEEE/ACM Transactions on Networking 26(3), 1278-1291 (2018)

9. Yu, J., Ning, X., Sun, Y., Wang, S., Wang, Y.: Constructing a self-stabilizing CDS with bounded diameter in wireless networks under SINR. In: IEEE INFOCOM, pp. 1-9 (2017)

10. Zhang, X., Yu, J., Li, W., Cheng, X., Yu, D., Zhao, F.: Localized algorithms for Yao graph-based spanner construction in wireless networks under SINR. IEEE/ACM Transactions on Networking 25(4), 2459-2472 (2017)

11. Tian, X., Yu, J., Ma, L., Li, G., Cheng, X.: Distributed deterministic broadcasting algorithms under the SINR model. In: IEEE INFOCOM, pp. 1-9 (2016)

12. Chiang, M., Hande, P., Lan, T., Tan, C.W., et al.: Power control in wireless cellular networks. Foundations and Trends $\AA$ in Networking 2(4), 381-533 (2008)

13. Huang, B., Yu, J., Yu, D., Ma, C.: SINR based Maximum Link Scheduling with Uniform Power in Wireless Sensor Networks. KSI Transactions on Internet \& Information Systems 8(11) (2014)

14. Luo, Z.-Q., Zhang, S.: Dynamic spectrum management: Complexity and duality. IEEE Journal of Selected Topics in Signal Processing 2(1), $57-73$ (2008)

15. Hassan, N.U., Yuen, C., Saeed, S., Zhang, Z.: Power control for sum-rate maximization on interference channels under sum power constraint. IEEE Transactions on Vehicular Technology 64(2), 593-609 (2014)

16. Liang, F., Shen, C., Yu, W., Wu, F.: Power control for interference management via ensembling deep neural networks. In: IEEE/CIC International Conference on Communications in China (ICCC), pp. 237-242 (2019)
17. Chiang, M., Tan, C.W., Palomar, D.P., O'neill, D., Julian, D.: Power control by geometric programming. IEEE Transactions on Wireless Communications 6(7), 2640-2651 (2007)

18. Qian, L.P., Zhang, Y.J., Huang, J.: MAPEL: Achieving global optimality for a non-convex wireless power control problem. IEEE Transactions on Wireless Communications 8(3), 1553-1563 (2009)

19. Razaviyayn, M., Hong, M., Luo, Z.-Q.: A unified convergence analysis of block successive minimization methods for nonsmooth optimization. SIAM Journal on Optimization 23(2), 1126-1153 (2013)

20. Mehanna, O., Huang, K., Gopalakrishnan, B., Konar, A., Sidiropoulos, N.D.: Feasible point pursuit and successive approximation of non-convex qcqps. IEEE Signal Processing Letters 22(7), 804-808 (2014)

21. Yang, Y., Pesavento, M.: A unified successive pseudoconvex approximation framework. IEEE Transactions on Signal Processing 65(13), 3313-3328 (2017)

22. Hong, M., Li, Q., Liu, Y.-F.: Decomposition by successive convex approximation: A unifying approach for linear transceiver design in heterogeneous networks. IEEE Transactions on Wireless Communications 15(2), 1377-1392 (2015)

23. Scutari, G., Sun, Y.: Parallel and distributed successive convex approximation methods for big-data optimization. In: Multi-agent Optimization, pp. 141-308 (2018)

24. Razaviyayn, M., Sanjabi, M., Luo, Z.-Q.: A stochastic successive minimization method for nonsmooth nonconvex optimization with applications to transceiver design in wireless communication networks. Mathematical Programming 157(2), 515-545 (2016)

25. Kaleva, J., Tölli, A., Juntti, M.: Decentralized sum rate maximization with QoS constraints for interfering broadcast channel via successive convex approximation. IEEE Transactions on Signal Processing 64(11) 2788-2802 (2016)

26. Yang, Y., Pesavento, M., Chatzinotas, S., Ottersten, B.: Energy Efficiency Optimization in MIMO Interference Channels: A Successive Pseudoconvex Approximation Approach. IEEE Transactions on Signal Processing 67(15), 4107-4121 (2019)

27. Sardellitti, S., Scutari, G., Barbarossa, S.: Joint cell selection and radio resource allocation in mimo small cell networks via successive convex approximation. In: 2014 IEEE International Conference on Acoustics, Speech and Signal Processing (ICASSP), pp. 850-854 (2014)

28. Wang, T., Vandendorpe, L.: Successive convex approximation based methods for dynamic spectrum management. In: IEEE International Conference on Communications (ICC), pp. 4061-4065 (2012)

29. Wang, H., Chen, J., Ding, G., Wang, S.: D2D Communications Underlaying UAV-Assisted Access Networks. IEEE Access 6, 46244-46255 (2018)

30. Wang, H., Ding, G., Wang, J., Wang, S., Wang, L.: Power control for multiple interfering D2D communications underlaying cellular networks: An approximate interior point approach. In: 2017 IEEE International Conference on Communications Workshops (ICC Workshops), pp. 1346-1351 (2017)

31. Foschini, G.J., Miljanic, Z.: A simple distributed autonomous power control algorithm and its convergence 42(4), 641-646 (1993)

32. Mitra, D.: An Asynchronous Distributed Algorithm for Power Control in Cellular Radio Systems. In: Holtzman, J.M., Goodman, D.J. (eds.) Wireless and Mobile Communications. The Springer International Series in Engineering and Computer Science (Communications and Information Theory), vol. 277, pp. 177-186. Springer, Boston, MA (1994)

33. Tae-Suk Kim, Seong-Lyun Kim: Random power control in wireless ad hoc networks. IEEE Communications Letters 9(12), 1046-1048 (2005)

34. Malekshan, K.R., Zhuang, W.: Joint scheduling and transmission power control in wireless ad hoc networks. IEEE Transactions on Wireless Communications 16(9), 5982-5993 (2017)

35. Shi, Y., Mallik, R.K., Letaief, K.B.: Randomized power control for two-hop interference channels. IEEE Communications Letters 14(12), 1128-1130 (2010)

36. Behzad, A., Rubin, I., Hsu, J.: On the performance of randomized power control algorithms in multiple access wireless networks. In: IEEE Wireless Communications and Networking Conference, 2005, vol. 2, pp. 707-7112 (2005)

37. Wei, W., Song, Z., Zhang, T., Zeng, Z.: An exponential power 
allocation of one-side interference channel with relay. In: International Symposium on Wireless Personal Multimedia Communications (2011)

38. Al-Shatri, H., Weber, T.: Optimizing power allocation in interference channels using DC programming. In: 8th International Symposium on Modeling and Optimization in Mobile, Ad Hoc, and Wireless Networks, pp. 360-366 (2010)

39. Etkin, R., Parekh, A., Tse, D.: Spectrum sharing for unlicensed bands. IEEE Journal on Selected Areas in Communications 25(3), 517-528 (2007)

40. Zhou, L., Ruttik, K., Tirkkonen, O., Berry, R.: Interference canceling power control games in gaussian interference channels. In: 2013 IEEE International Symposium on Information Theory, pp. 1112-1116 (2013)

41. Gjendemsjø, A., Gesbert, D., Øien, G.E., Kiani, S.G.: Binary power control for sum rate maximization over multiple interfering links. IEEE Transactions on Wireless Communications 7(8), 3164-3173 (2008)

42. Eriksson, K., Shi, S., Vucic, N., Schubert, M., Larsson, E.G.: Globally optimal resource allocation for achieving maximum weighted sum rate. In: IEEE Global Telecommunications Conference (GLOBECOM), pp. $1-6(2010)$

43. Kha, H.H., Tuan, H.D., Nguyen, H.H.: Fast global optimal power allocation in wireless networks by local dc programming. IEEE Transactions on Wireless Communications 11(2), 510-515 (2011)

44. Boyd, S., Grant, M.: The CVX Users Guide, (2018)

45. Zhang, H., Dong, A., Jin, S., Yuan, D.: Joint transceiver and power splitting optimization for multiuser MIMO SWIPT under MSE QoS constraints. IEEE Transactions on Vehicular Technology 66(8), 7123-7135 (2017)

46. Boyd, S., Parikh, N., Chu, E., Peleato, B., Eckstein, J.: Distributed Optimization and Statistical Learning Via the Alternating Direction Method of Multipliers, (2011)

47. Cadambe, V.R., Jafar, S.A.: Interference alignment and degrees of freedom of the $k$-user interference channel. IEEE Transactions on Information Theory 54(8), 3425-3441 (2008)

Figures

Figure 1 System model of the $\boldsymbol{K}$-user SISO IC network. There are $\mathcal{K}$ pairs of transceivers with single antenna in the network. The signal of a user constitutes interference to other receivers. The solid lines represent the signal links of the users and dashed lines denote the interference links.

Figure 2 Convergence of the SCA-based algorithm with $\left\{p_{k}^{0}=1.1 p_{k}^{m i n}, \forall k \in \mathcal{K}\right\}$. The optimized power and achieved rate versus number of iterations are plotted when the proposed SCA-based algorithm is initialized from a point near the minimum required power values, which are set as $p_{1}^{\text {min }}=10 \mathrm{~dB}, p_{2}^{\text {min }}=8 \mathrm{~dB}$ and $p_{3}^{\text {min }}=6 \mathrm{~dB}$, respectively. The curves show that the proposed algorithm converges after 6 iterations.

\section{Figure 3 Convergence of the SCA-based algorithm with} $\left\{p_{k}^{0}=0.98 p_{k}^{\max }, \forall k \in \mathcal{K}\right\}$. The optimized power and achieved rate versus number of iterations are plotted when the proposed SCA-based algorithm is initialized from a point near the maximum allowed power values, which are set as $p_{1}^{\max }=20 \mathrm{~dB}, p_{2}^{\max }=15 \mathrm{~dB}$ and $p_{3}^{\max }=10 \mathrm{~dB}$, respectively The curves show that the proposed algorithm converges after 4 iterations.
Figure 4 Convergence of the SCA-based algorithm with $p_{1}^{0}=84.8070, p_{2}^{0}=29.6569, p_{3}^{0}=\mathbf{7 . 8 6 2 2}$. The optimized power and achieved rate versus number of iterations are plotted when the proposed SCA-based algorithm is initialized from a point randomly generated within the range of $\left(p_{k}^{\min }, p_{k}^{\max }\right)$, which are set as $p_{1}^{\text {min }}=10 \mathrm{~dB}, p_{2}^{\min }=8 \mathrm{~dB}$ and $p_{3}^{\min }=6 \mathrm{~dB}, p_{1}^{\max }=20 \mathrm{~dB}, p_{2}^{\max }=15 \mathrm{~dB}$ and $p_{3}^{\max }=10 \mathrm{~dB}$, respectively. The curves show that the proposed algorithm converges after 3 iterations.

Figure 5 Comparison of the optimized transmit powers of the proposed SCA-based algorithm and the GP-based algorithm. The results show that the proposed SCA-based algorithm allocates the maximum allowed power to user 1 and the minimum required powers to the user 2 and user 3 , respectively. While the powers allocated by the GP-based algorithm fall in the ranges between the minimum required values and maximum allowed values.

Figure 6 Comparison of the achieved data rates of the proposed SCA-based algorithm and the GP-based algorithm. The results show that the sum rate achieved by the proposed SCA-based algorithm is greater than $3 \mathrm{bps} / \mathrm{Hz}$, which is much higher than that achieved by the GP-based algorithm.

Figure 7 Sum rate achieved by the SCA-based algorithm over 100 randomly feasible initial points. The network is configured as $p_{k}^{\max }=20 \mathrm{~dB}$ and $p_{k}^{\min }=0, \forall k \in \mathcal{K}$, This figure shows that three solutions can be obtained by the proposed SCA-based algorithm for the considered example. This means that the algorithm may be stuck in a locally optimal solution.

Figure 8 Sum rate versus the pseudo signal to noise ratio SNR ${ }^{\max }$ for different power control schemes. The result of the proposed SCA-based algorithm is obtained by selecting the best solution out of 10 times of simulations at each $\mathrm{SNR}^{\max }$. The results show that the proposed SCA-based algorithm achieves the best performance at any given SNR value. Specifically, at the high SNR regime, the proposed algorithm achieves similar performance as the greedy scheme, which activates only one user that has the strongest channel gain. While at the low SNR regime, the proposed algorithm performs similarly to the GP-based algorithm and superior to the greedy algorithm. 
Figures

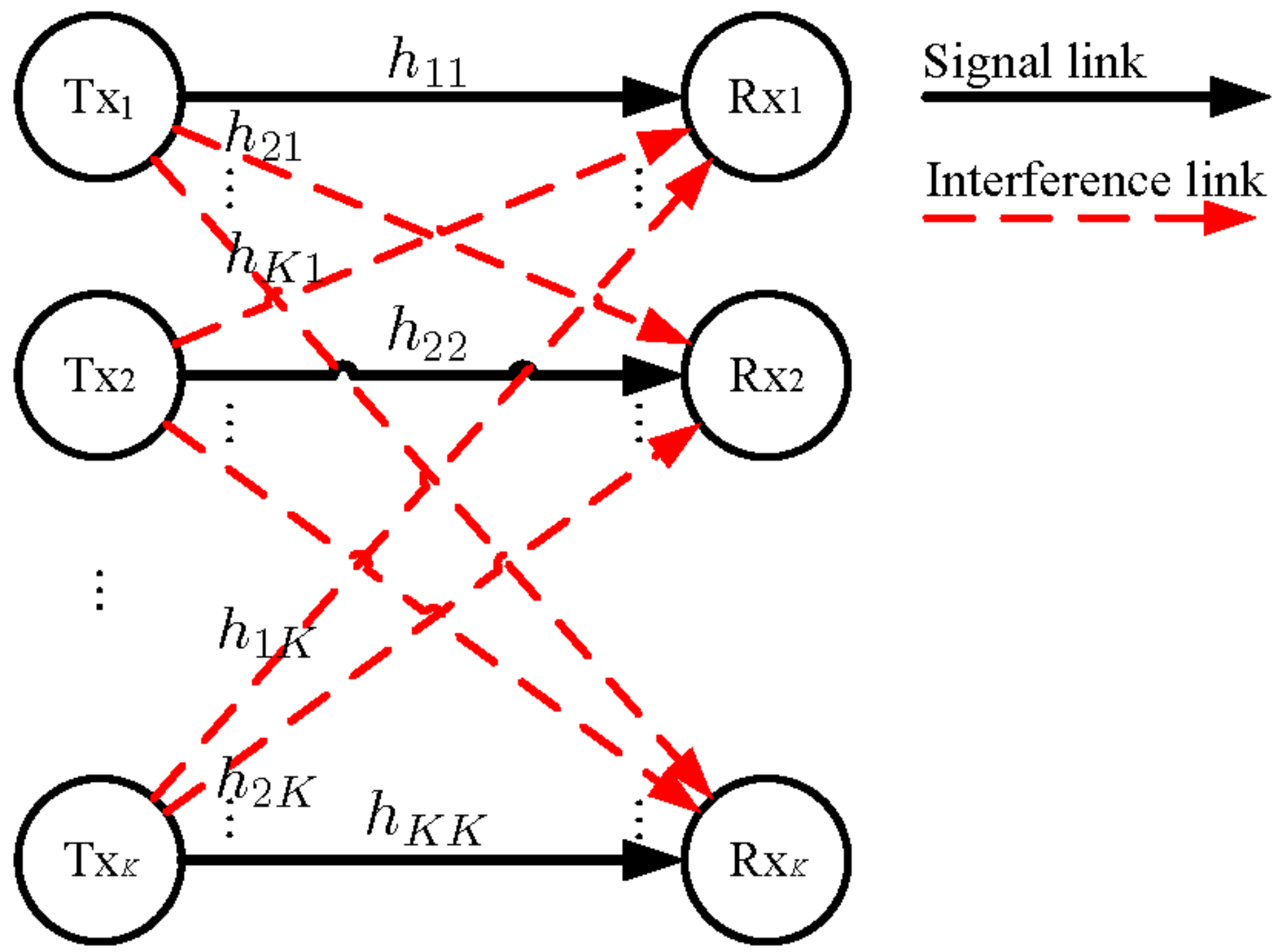

Figure 1

System model of the K-user SISO IC network. 


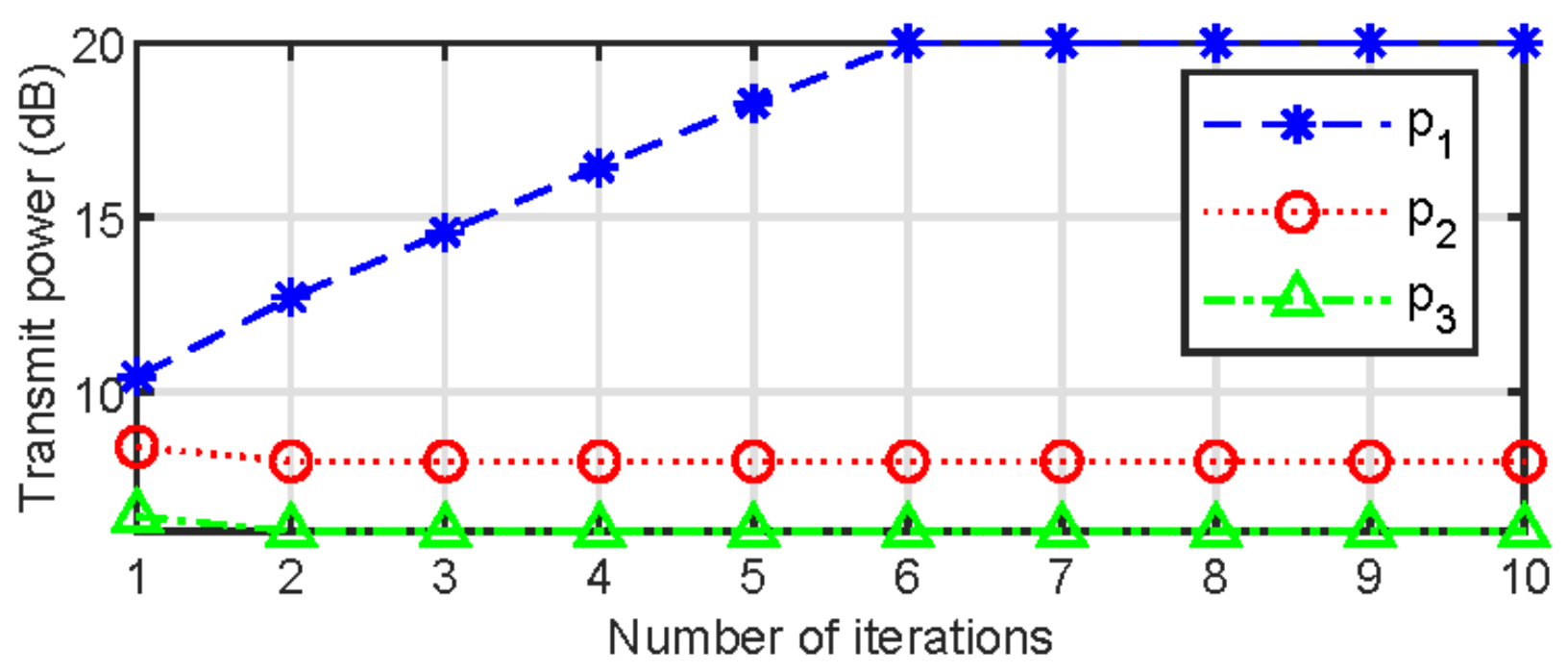

(a) Transmit power

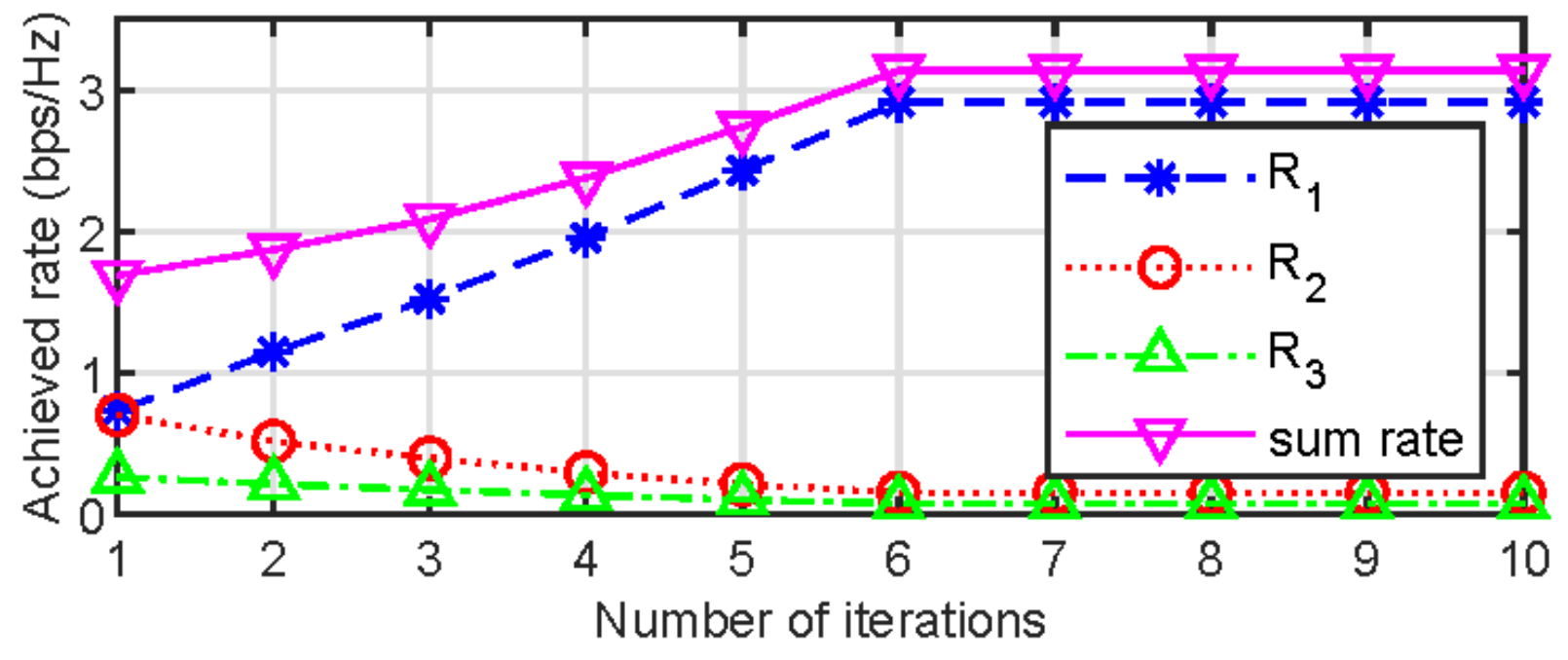

(b) Achieved rate

Figure 2

Convergence of the SCA-based algorithm with $\{\mathrm{p} 0 \mathrm{k}=1: 1 \mathrm{pkmin}, \varangle \mathrm{k} ; 8 \mathrm{k} 2 \otimes \mathrm{K}\}$ 


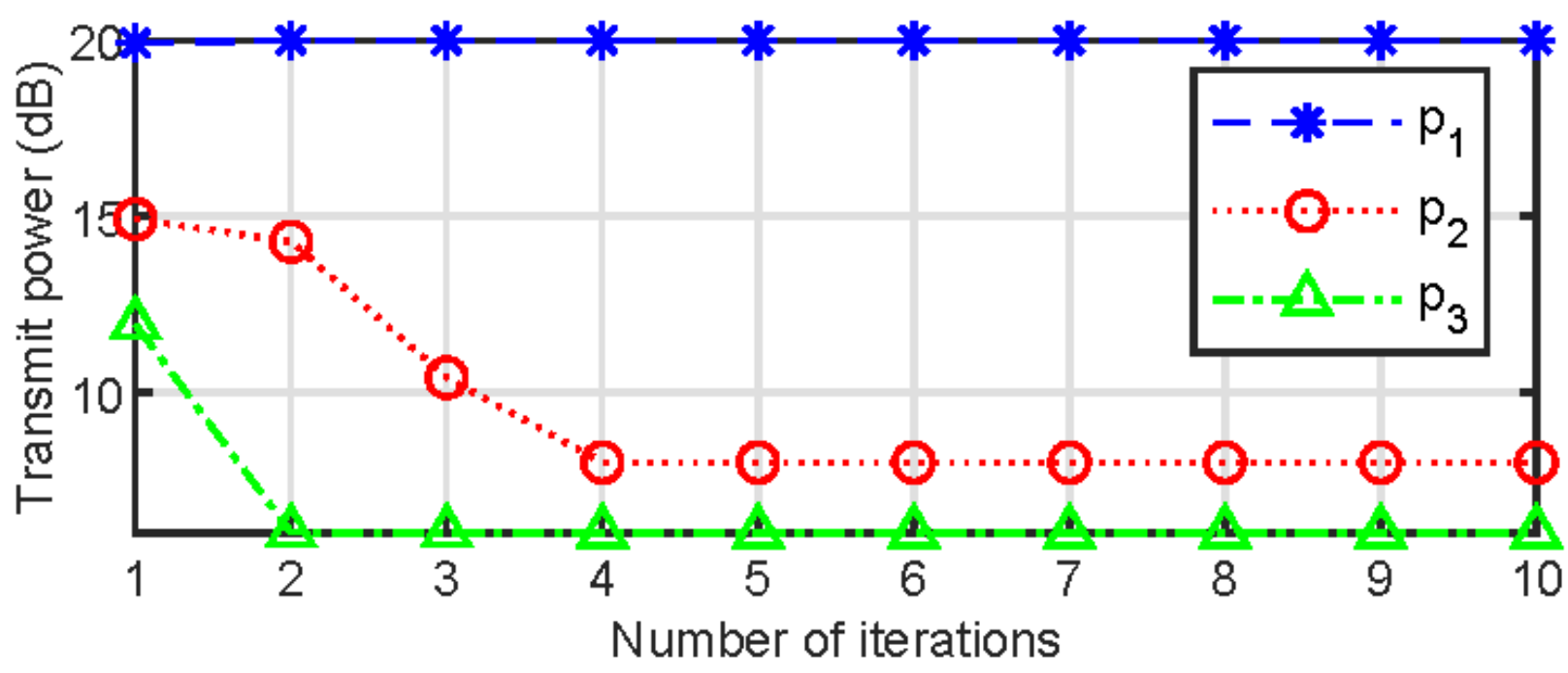

(a) Transmit power

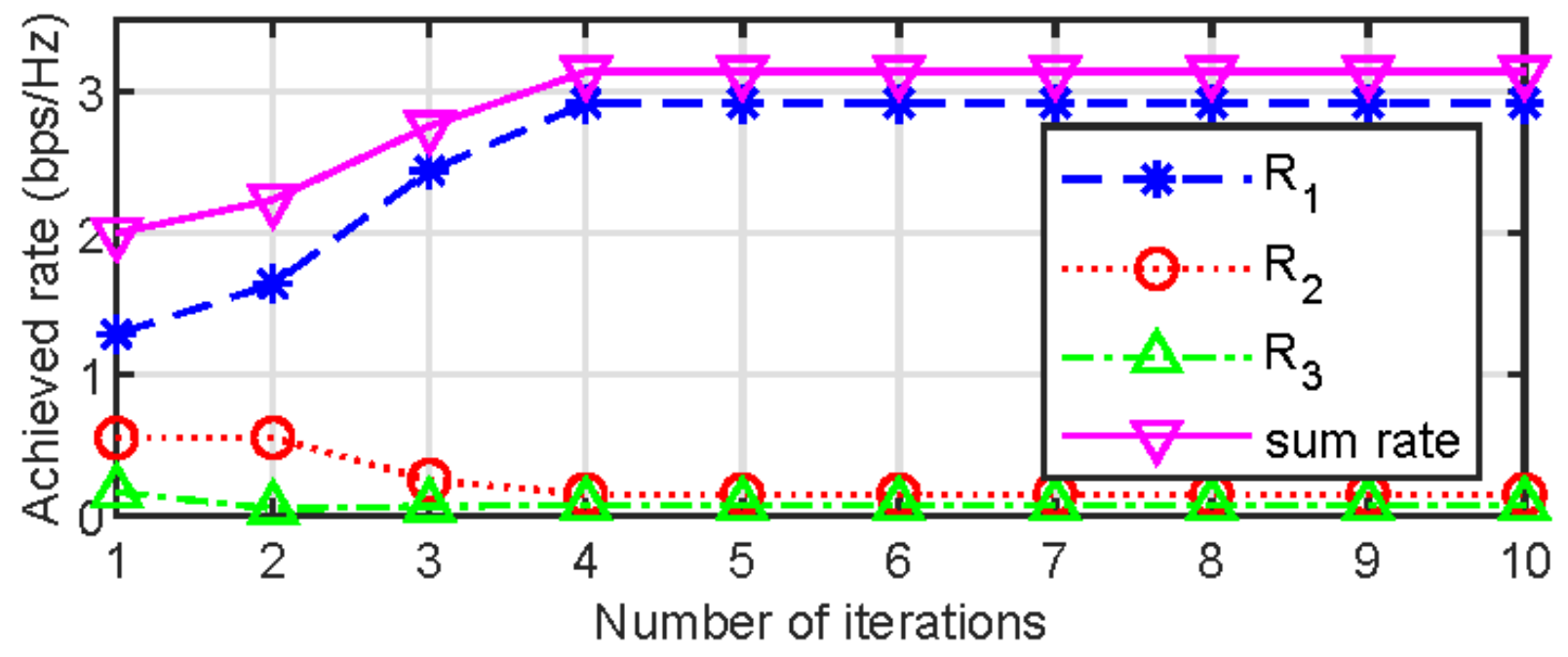

(b) Achieved rate

Figure 3

Convergence of the SCA-based algorithm with $\{p 0 k=0: 98 p m a x k ; \varangle k \otimes K\}$ 


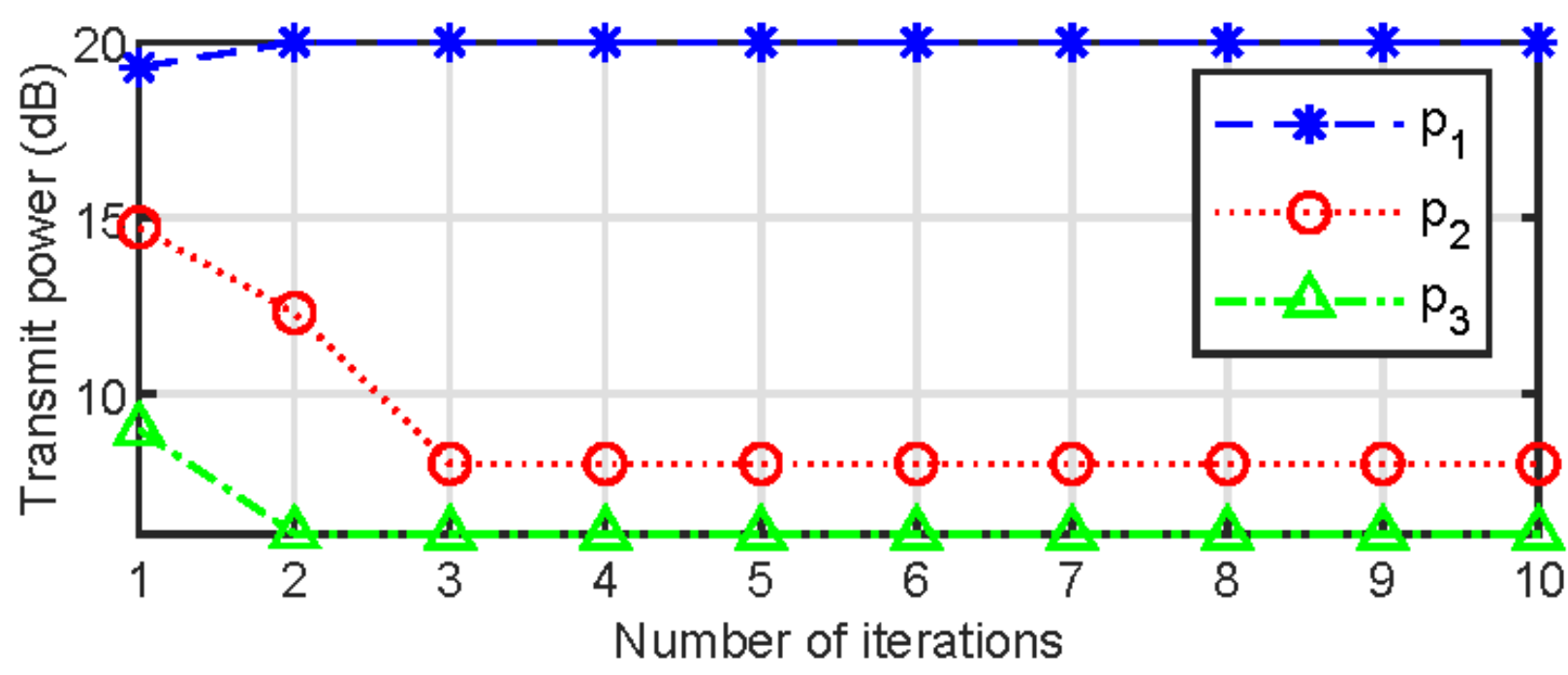

(a) Transmit power

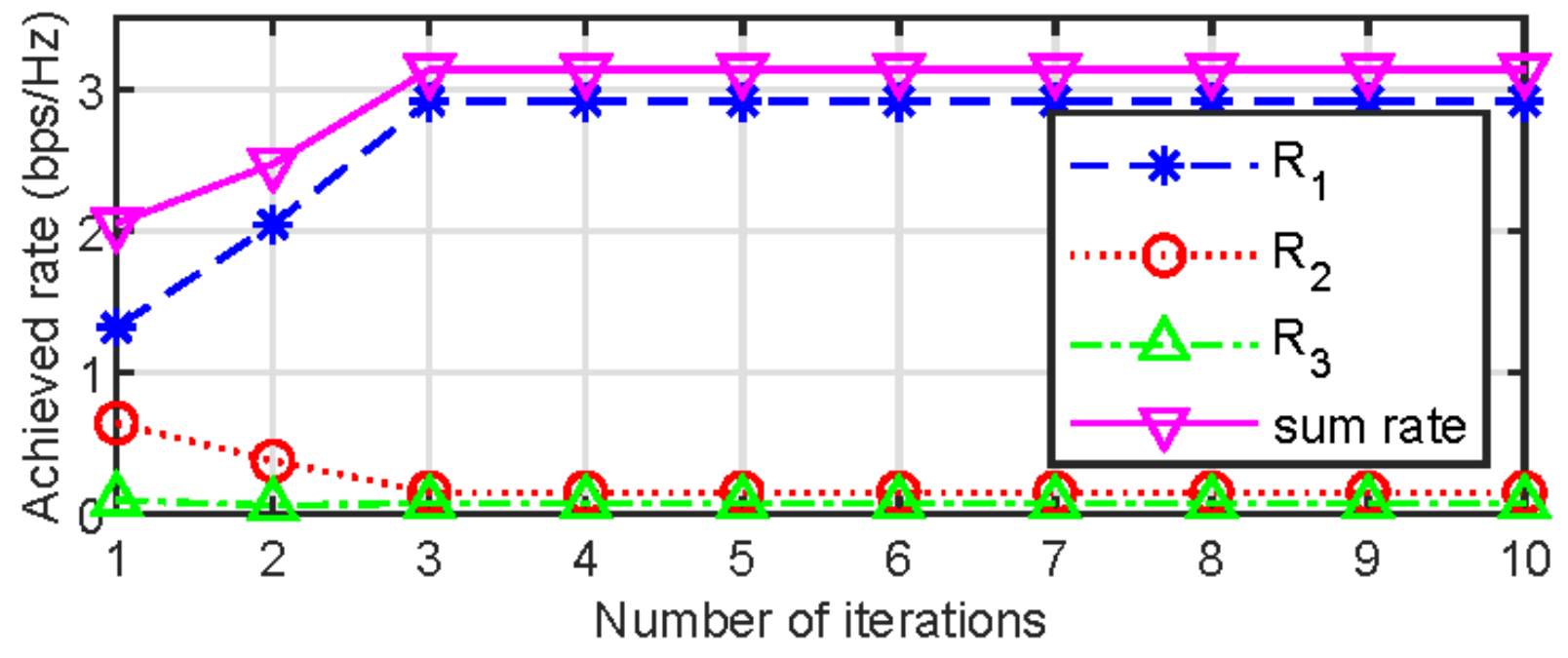

(b) Achieved rate

Figure 4

Convergence of the SCA-based algorithm with $\{p 01=84: 8070 ; p 02=29: 6569 ; p 03=7: 8622\}$. 


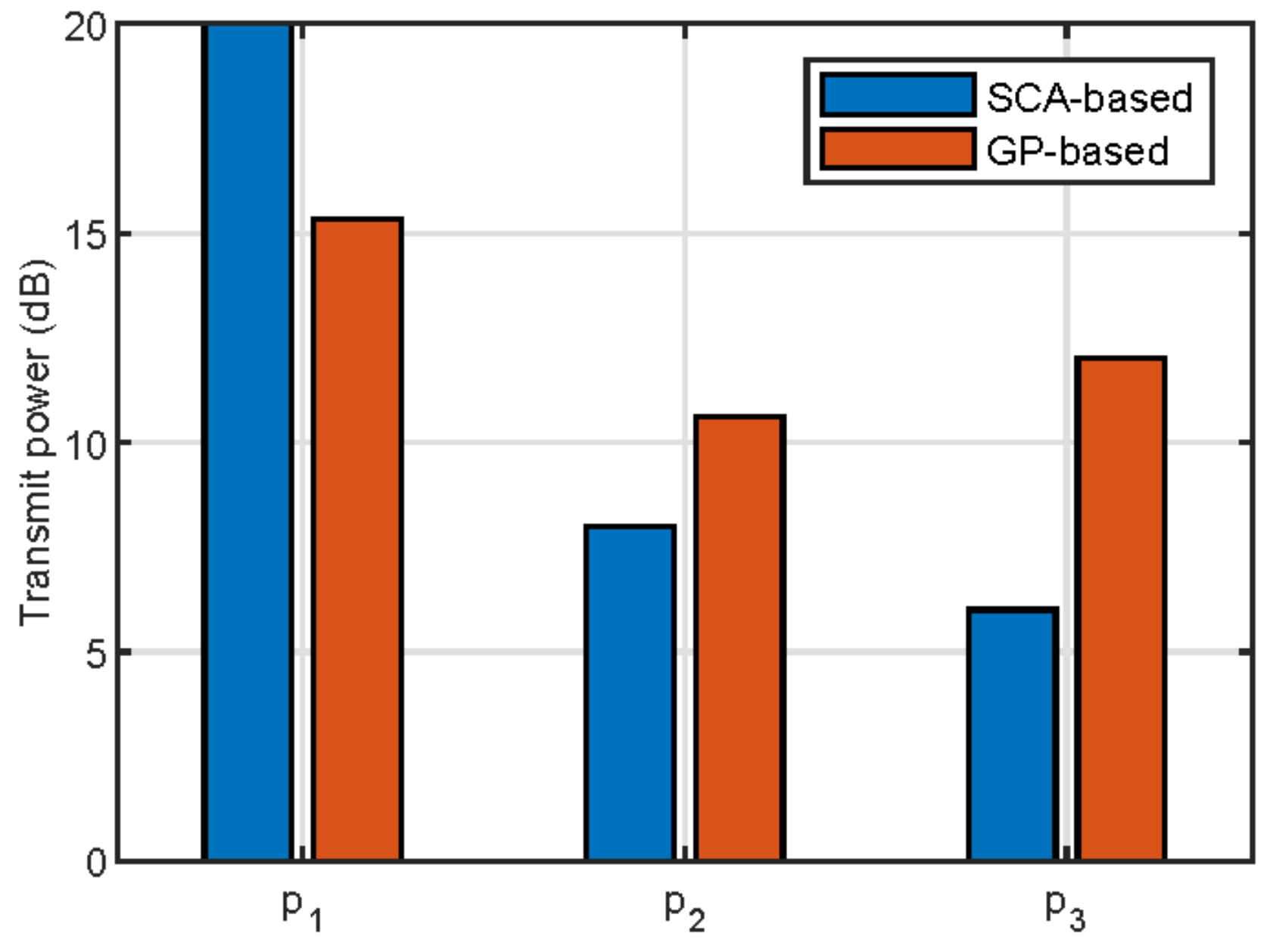

Figure 5

Comparison of the optimized transmit powers of the proposed SCA-based algorithm and the GP-based algorithm. 


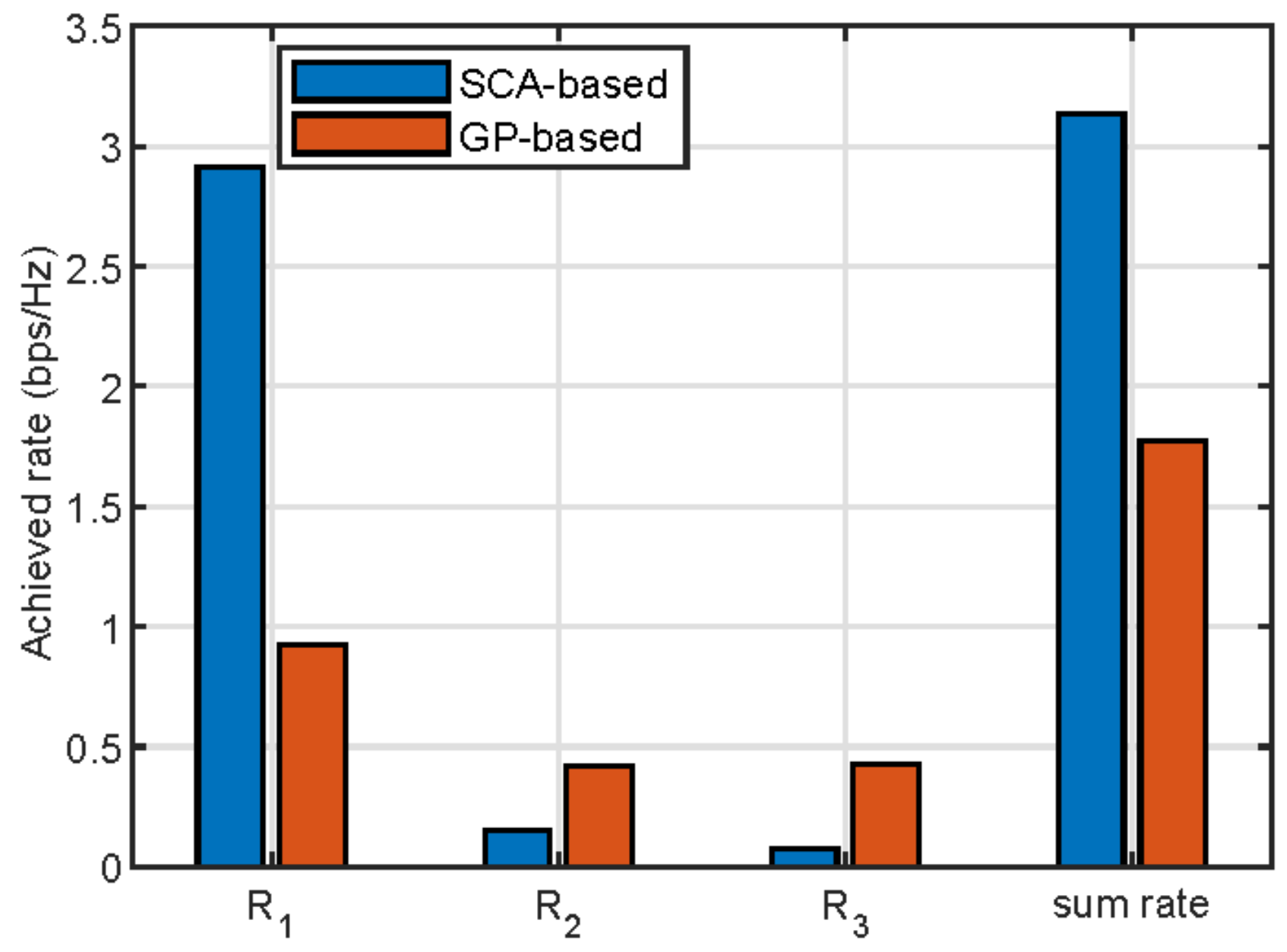

Figure 6

Comparison of the achieved data rates of the proposed SCA-based algorithm and the GP-based algorithm. 


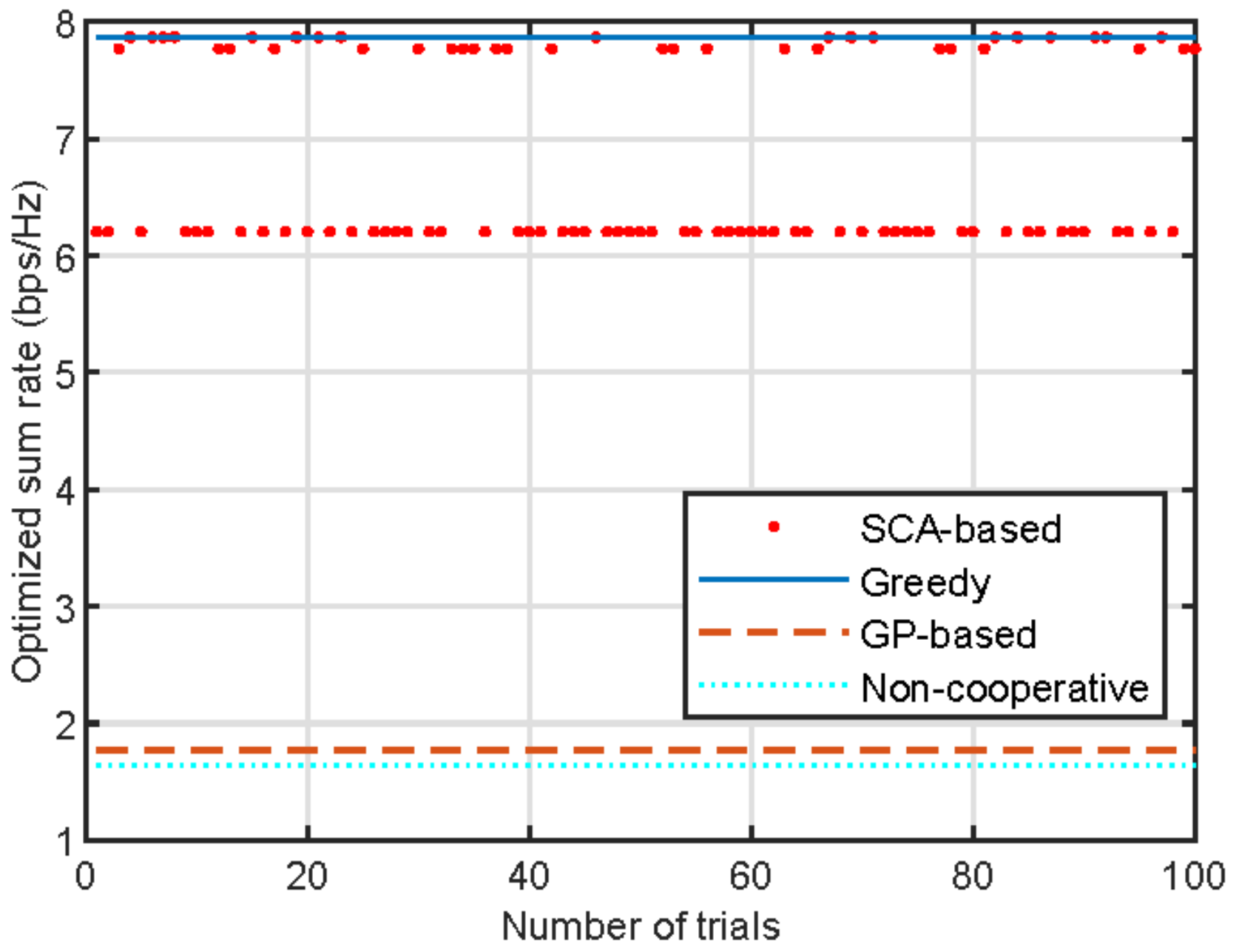

Figure 7

Sum rate achieved by the SCA-based algorithm over 100 randomly feasible initial points with pmaxk = $20 \mathrm{~dB}$ and pmink $=0, \nabla \mathrm{k} \otimes \mathrm{K}$ 


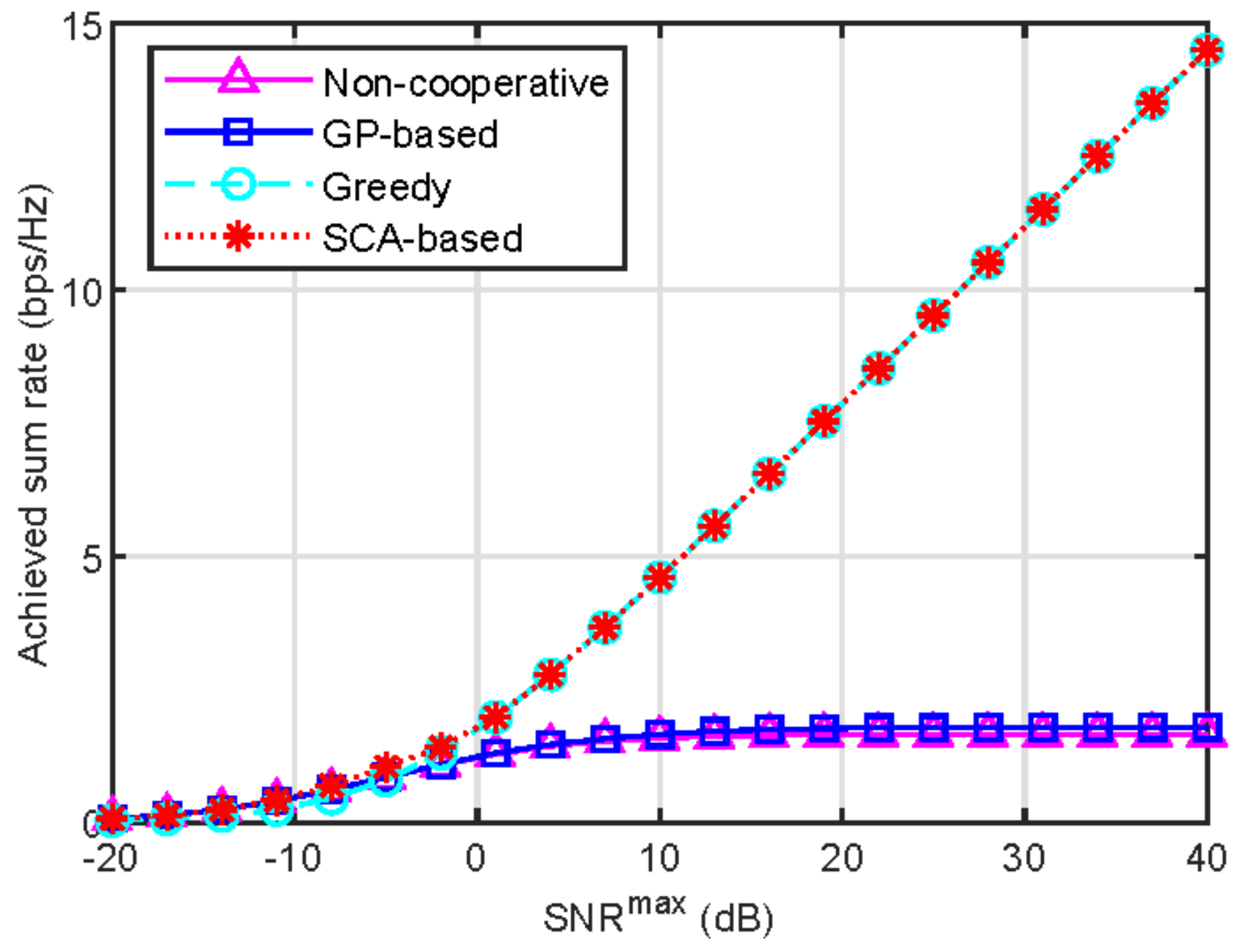

Figure 8

Sum rate versus the pseudo signal to noise ratio SNRmax for different power control schemes with pmaxk $=20 \mathrm{~dB}$ and pmink $=0$, $\nabla \mathrm{k} \otimes \mathrm{K}$ 